(C) ACM 2005. This is the author's version of the work. It is posted here by permission of ACM for your personal use. Not for redistribution. The definitive version was published in Volume 6 Issue 2, Pages 295-327 , 2005, https://doi.org/10.1145/1055686.1055690

\title{
Comparisons and Computation of Well-founded Semantics for Disjunctive Logic Programs
}

\author{
Kewen Wang* \\ School of Computing and Information Technology \\ Griffith University, QLD 4111, Australia \\ k.wang@cit.gu.edu.au \\ http://www.cit.gu.edu.au/ s2107085/ \\ Lizhu Zhou \\ Department of Computer Science and Technology \\ Tsinghua University, Beijing \\ deszlz@tsinghua.edu.cn
}

\begin{abstract}
Much work has been done on extending the well-founded semantics to general disjunctive logic programs and various approaches have been proposed. However, these semantics are different from each other and no consensus is reached about which semantics is the most intended. In this paper we look at disjunctive well-founded reasoning from different angles. We show that there is an intuitive form of the well-founded reasoning in disjunctive logic programming which can be characterized by slightly modifying some exisitng approaches to defining disjunctive well-founded semantics, including program transformations, argumentation, unfounded sets (and resolution-like procedure). We also provide a bottom-up procedure for this semantics. The significance of our work is not only in clarifying the relationship among different approaches, but also shed some light on what is an intended well-founded semantics for disjunctive logic programs.
\end{abstract}

\section{Introduction}

The importance of representing and reasoning about disjunctive information has been addressed by many researchers. As pointed out in [4], the related application domains include reasoning by cases, legal reasoning, diagnosis, natural language understanding and conflict resolving in multiple inheritance. Disjunctive logic programming (DLP) is widely believed to be a suitable tool for

\footnotetext{
* This work was done while the second author was with the University of Potsdam.
} 
formalizing disjunctive reasoning and it has received extensive studies in recent years, e. g. [1; 2. 9, 11. Since DLP admits both default negation and disjunction, the issue of finding a suitable semantics for disjunctive programs is more difficult than it is in the case of normal (i. e. non-disjunctive) logic programs. Usually, skepticism and credulism represent two major semantic intuitions for knowledge representation in artificial intelligence. The wellfounded semantics [16] is a formalism of skeptical reasoning in normal logic programming while the stable semantics $[8]$ formalizes credulous reasoning. Recently, considerable effort has been paid to generalize these two semantics to disjunctive logic programs. However, the task of generalizing the well-founded model to disjunctive programs has been proven to be complex. There have been various proposals for defining the well-founded semantics for general disjunctive logic programs [11]. As argued by some authors (for instance [4, 13, 17l), each of the previous versions of the disjunctive well-founded semantics bears its own drawbacks. Moreover, no consensus is reached about what constitutes an intended well-founded semantics for disjunctive logic programs. The semantics D-WFS [3, 4], STATIC [13] and WFDS [17] are among the most recent approaches to defining disjunctive well-founded semantics. D-WFS is based on a series of abstract properties and it is the weakest (least) semantics that is invariant under a set of program transformations. STATIC has its root in autoepistemic logic and is based on the notion of static expansions for belief theories. The semantics STATIC $(P)$ for a disjunctive program $P$ is defined as the least static expansion of $P_{\mathrm{AEB}}$ where $P_{\mathrm{AEB}}$ is the belief theory corresponding to $P$. The basic idea of WFDS is to transform $P$ into an argumentation framework and $\operatorname{WFDS}(P)$ is specified by the least acceptable hypothesis of $P$. Although these semantics stem from very different intuitions, all of them share a number of attractive properties. For instance, each of these semantics extends both the well-founded semantics [16 for normal logic programs and the generalized closed world assumption (GCWA) [12] for positive disjunctive programs (i. e. without default negation); each of these semantics is consistent and provides approximation to the disjunctive stable semantics (i.e. a literal derived under the well-founded semantics is also derivable from any stable model).

However, the problem of comparing different approaches to defining disjunctive wellfounded semantics is rarely investigated. A good starting point is [5] in which it is proven that D-WFS is equivalent to a restricted version of STATIC. But the relation of D-WFS to the argumentation-based semantics and unfounded sets is as yet unclear. More importantly, it is an open question whether there is a disjunctive well-founded semantics that can be characterized by all of these approaches.

In this paper, we intuitively (and slightly) modify some existing semantics and report further equivalence results:

1. As we will see in Section 5, the transformation-based semantics D-WFS is different from WFDS and seems a little too skeptical. The reason is that the program transformations in Brass and Dix's set $\mathbf{T}_{\mathrm{WFS}}$ are unable to reduce the rule head if we do not remove a rule from the disjunctive program. Interestingly, this problem is related to the famous GCWA (Generalized Closed World Assumption) [12]. Based on this observation, we introduce a new program transformation called the Elimination of s-implications, which naturally extends the Elimination of nonminimal rules in [4]. 
We define a new transformation-based semantics, denoted D-WFS*, as the weakest semantics that allows the Elimination of s-implications and the program transformations in $\mathbf{T}_{\mathrm{WFS}}$ except for the Elimination of nonminimal rules. This semantics naturally extends D-WFS and enjoys all the important properties that have been proven for D-WFS. An important result in this paper is that WFDS is equivalent to D-WFS*, which establishes a precise relationship between argumentation-based approach and transformation-based approach in disjunctive logic programming.

2. The notion of unfounded sets is well-known in logic programming. It was first employed by [16] to define the well-founded semantics for normal logic programs and then to characterize other semantics including the stable models [8] and partial stable models [15]. This notion has been generalized from normal to disjunctive logic programs [7; 10]. Although a form of disjunctive well-founded semantics is investigated in [10, the generalized unfounded sets are mainly used to characterize partial stable models for disjunctive programs. Moreover, their notions are not appropriate for defining a disjunctive well-founded semantics as we will see in Section 7 . One reason for this is that their notions are defined only for interpretations (i. e. consistent sets of literals) rather than for model states (i. e. sets of disjunctions of literals) [11]. Thus, we further generalize the notion of unfounded sets to model states. The resulting disjunctive well-founded semantics, denoted U-WFS, behaves more intuitive and actually we show that it is also equivalent to WFDS.

3. We develop a bottom-up evaluation procedure for WFDS (equivalently, for D-WFS*, U-WFS) in a similar way as in [4]. Specifically, for each disjunctive program $P$, it can be gradually transformed into a normal form called strong residual program $\operatorname{res}^{*}(P)$ by our elementary program transformations. We show that the semantics WFDS $(P)$ can be directly read out from the strong residual program: if there is no rule head containing an atom $p$, then not $p \in \operatorname{WFDS}(P)$; if there is a rule of the form $A \leftarrow$ in the strong residual program, then $A \in \operatorname{WFDS}(P)$. That is, our bottom procedure is sound and complete with respect to WFDS.

Moreover, in [18] we have developed a top-down procedure D-SLS Resolution which is sound and complete with respect to WFDS. D-SLS naturally extends both SLS-resolution [14] (for normal logic program) and SLI-resolution [11] (for disjunctive programs without default negation).

Altogether we obtain the following equivalence results:

$$
\mathrm{WFDS} \equiv \mathrm{D}-W F S^{*} \equiv \mathrm{U}-\mathrm{WFS} \equiv \mathrm{D}-\mathrm{SLS} .
$$

We consider these results to be quite significant:

1. Our results clarify the relationship among several different approaches to defining disjunctive well-founded semantics, including argumentation-based, transformation-based, unfounded sets-based (and resolution-based approaches). 
2. Since the four semantics are based on very different intuitions, these equivalent characterizations in turn shed some light on what is an intended well-founded semantics for disjunctive logic programs.

3. The bottom-up query evaluation proposed in this paper paves a promising way for implementing disjunctive well-founded semantics.

The rest of this paper is arranged as follows. In Section 2 we recall some basic definitions and notation; we present in Section 3 a slightly restricted form of the well-founded semantics WFDS (we still denote WFDS). In Section 5 we introduce the program transformation Elimination of s-implications and then define the transformation-based semantics D-WFS*, which naturally extends D-WFS. In Section 6 , we first provide a bottom-up query evaluation for D-WFS* (equivalently, WFDS) and then prove the equivalence of D-WFS* and WFDS. Section 7 introduces a new notion of unfounded sets and defines the well-founded semantics U-WFS. We also show that U-WFS is equivalent to WFDS. Section 8 is our conclusion.

\section{Preliminaries}

We briefly review most of the basic notions used throughout this paper.

A disjunctive logic program is a finite set of rules of the form

$$
a_{1} \vee \cdots \vee a_{n} \leftarrow b_{1}, \ldots, b_{m}, \text { not } c_{1}, \ldots, \text { not } c_{t},
$$

where $a_{i}, b_{i}, c_{i}$ are atoms and $n>0$. The default negation 'not $a$ ' of an atom $a$ is called a negative literal.

In this paper we consider only propositional programs although some of the definitions and results hold for predicate logic programs.

For technical reasons, it should be stressed that the body of a rule is a set of literals rather than a multiset. For instance, $a \vee b \leftarrow c, c$ is not a rule in our sense while $a \vee b \leftarrow c$ is a rule. That is, we assume that any rule of a logic program has been simplified by eliminating repeated literals in both its head and body.

$P$ is a normal logic program if it contains no disjunctions.

If a rule of form (1) contains no negative body literals, it is called positive; $P$ is a positive program if every rule of $P$ is positive.

If a rule of form (11) contains no body atoms, it is called negative; $P$ is a negative program if every rule of $P$ is negative.

Following [4], we also say a negative rule $r$ is a conditional fact. That is, a conditional fact is of form $a_{1} \vee \cdots \vee a_{n} \leftarrow$ not $c_{1}, \cdots$, not $c_{t}$, where $a_{i}$ and $c_{j}$ are (ground) atoms for $1 \leq k \leq n$ and $0 \leq j \leq t$.

For a rule $r$ of form (11), $\operatorname{body}(r)=b o d y^{+}(r) \cup b o d y^{-}(r)$ where $b o d y^{+}(r)=\left\{b_{1}, \ldots, b_{m}\right\}$ and $\operatorname{body}^{-}(r)=\left\{\right.$ not $c_{1}, \ldots$, not $\left.c_{t}\right\}$; head $(r)=a_{1} \vee \cdots \vee a_{n}$. When no confusion is caused, we also use head $(r)$ to denote the set of atoms in head $(r)$. For instance, $a \in$ head $(r)$ means that $a$ appears in the head of $r$. If $X$ is a set of atoms, head $(r)-X$ is the disjunction obtained 
from head $(r)$ by deleting the atoms in $X$. The set head $(P)$ consists of all atoms appearing in rule heads of $P$.

In the sequel, we will use the capital letters $A, B, C$ to represent both disjunctions or sets of atoms (in case there is confusion, we will explicitly claim their scopes).

As usual, $B_{P}$ is the Herbrand base of disjunctive logic program $P$, that is, the set of all (ground) atoms in $P$. A positive (negative) disjunction is a disjunction of atoms (negative literals) of $P$. A pure disjunction is either a positive one or a negative one.

The disjunctive base of $P$ is $\mathrm{DB}_{P}=\mathrm{DB}_{P}^{+} \cup \mathrm{DB}_{P}^{-}$where $\mathrm{DB}_{P}^{+}$is the set of all positive disjunctions in $P$ and $\mathrm{DB}_{P}^{-}$is the set of all negative disjunctions in $P$. If $A$ and $B=A \vee A^{\prime}$ are two disjunctions, then we say $A$ is a sub-disjunction of $B$, denoted $A \subseteq B$. $A \subset B$ means $A \subseteq B$ but $A \neq B$

A model state of a disjunctive program $P$ is a subset of $\mathrm{DB}_{P}$.

A model state $S$ is inconsistent if at least one of the following two conditions holds:

1. There is a positive disjunction $a_{1} \vee \cdots \vee a_{n} \in S(n \geq 1)$ such that not $a_{i} \in S$ for all $1 \leq i \leq n$; or

2. There is a negative disjunction not $a_{1} \vee \cdots \vee$ not $a_{n} \in S(n \geq 1)$ such that $a_{i} \in S$ for all $1 \leq i \leq n$.

Otherwise, we say that $S$ is a consistent model state.

Usually, a well-founded semantics for a disjunctive logic program is defined by a (consistent) model state.

If $E$ is an expression (a set of literals, a disjunction or a set of disjunctions), atoms $(E)$ denotes the set of all atoms appearing in $E$.

For simplicity, we assume that all model states are closed under implication of pure disjunctions. That is, for any model state $S$, if $A$ is a sub-disjunction of a pure disjunction $B$ and $A \in S$, then $B \in S$. For instance, if $S=\{a, b \vee c\}$, then we implicitly assume that $a \vee b \vee c \in S$.

Given a model state $S$ and a pure disjunction $A$, we also say $A$ is satisfied by $S$, denoted $S \models A$, if $A \in S$.

We assume that all disjunctions have been simplified by deleting the repeated literals. For example, the disjunction $a \vee b \vee b$ is actually the disjunction $a \vee b$.

For any set $S$ of disjunctions, the canonical form of $S$ is defined as $\operatorname{can}(S)=\{A \in S \mid$ there is no disjunction $A^{\prime} \in S$ s. t. $\left.A^{\prime} \subset A\right\}$.

We recall that the least model state of a positive disjunctive program $P$ is defined as

$$
\operatorname{ms}(P)=\left\{A \in \mathrm{DB}_{P}^{+} \mid P \vdash A\right\}
$$

Here $\vdash$ is the inference relation of the classical propositional logic.

Given a positive disjunctive program $P, \operatorname{can}(\mathrm{ms}(P))$ can also be equivalently characterized by the least fixpoint of the immediate consequence operator $T_{P}^{G}$ for $P$ (see [11] for details). 
Definition 1 Let $P$ be a positive disjunctive program and let $J$ be a subset of $D B_{P}^{+}$. The immediate consequence operator $T_{P}^{S}: 2^{D B_{P}^{+}} \rightarrow 2^{D B_{P}^{+}}$is defined as follows

$$
T_{P}^{S}(J)=\left\{\begin{array}{l|l}
A \in D B_{P}^{+} \mid \begin{array}{l}
\text { there exist a rule } A^{\prime} \leftarrow b_{1}, \ldots, b_{m} \\
\text { in } P \text { and } A_{1}, \ldots, A_{m} \in D B_{P}^{+} \text {such that } \\
\text { (1). }\left(b_{i} \vee A_{i}\right) \in J, \text { for all } i=1, \ldots, m ; \text { and } \\
(2) . \quad A=A^{\prime} \vee A_{1} \vee \cdots \vee A_{m}
\end{array}
\end{array}\right\}
$$

$T_{P}^{S}$ is actually the following hyperresolusion:

$$
\frac{b_{1} \vee A_{1} ; \ldots, b_{m} \vee A_{m} ; A^{\prime} \leftarrow b_{1}, \ldots, b_{m}}{A^{\prime} \vee A_{1} \vee \cdots \vee A_{m}}
$$

Note that we always remove repetitions of literals in each rule and/or disjunctions.

Define $T_{P}^{S} \uparrow 0=\emptyset$, and $T_{P}^{S} \uparrow(n+1)=T_{P}^{S}\left(T_{P}^{S} \uparrow n\right)$ for $n \geq 0$. Then we have the following result [1].

Theorem 2.1 Let $P$ be a positive disjunctive program. Then,

$$
\operatorname{can}(m s(P))=\operatorname{can}\left(\operatorname{lfp}\left(T_{P}^{S}\right)\right)=\operatorname{can}\left(T_{P}^{S} \uparrow \omega\right) .
$$

\section{Argumentation and well-founded semantics}

As illustrated in $[17]^{1}$, argumentation provides an unifying semantic framework for DLP. The basic idea of the argumentation-based approach for DLP is to translate each disjunctive logic program into an argument framework $\mathbf{F}_{P}=\left\langle P, \mathrm{DB}_{P}^{-}, \sim_{P}\right\rangle$. In that framework, an assumption of $P$ is a negative disjunction of $P$, and a hypothesis is a set of assumptions; $\sim_{P}$ is an attack relation among the hypotheses. An admissible hypothesis $\Delta$ is one that can attack every hypothesis which attacks it. The intuitive meaning of an assumption not $a_{1} \vee \cdots \vee$ not $a_{m}$ is that $a_{1} \wedge \cdots \wedge a_{m}$ can not be proven from the disjunctive program.

Given a hypothesis $\Delta$ of disjunctive program $P$, similar to the GL-transformation [8], we can easily reduce $P$ into another disjunctive program without default negation.

Definition 2 Let $\Delta$ be a hypothesis of disjunctive program $P$, then the reduct of $P$ with respect to $\Delta$ is the disjunctive program

$$
P_{\Delta}^{+}=\left\{\operatorname{head}(r) \leftarrow \operatorname{bod} y^{+}(r) \mid r \in P \text { and body }(r) \subseteq \Delta\right\} .
$$

It is obvious that $P_{\Delta}^{+}$is a positive disjunctive program (i .e. without default negation).

Example 3.1 Let $P$ be the following disjunctive program:

$$
\begin{aligned}
a & \leftarrow b, \text { not } c \\
b \vee c & \leftarrow \text { note } \\
b \vee c \vee d & \leftarrow
\end{aligned}
$$

\footnotetext{
${ }^{1}$ You et al in [19] also defined an argumentative extension to the disjunctive stable semantics. However, their framework does not lead to an intuitive well-founded semantics for DLP as the authors have observed.
} 
If $\Delta=\{$ not $c\}$, then $P_{\Delta}^{+}$is the positive program:

$$
\begin{aligned}
a & \leftarrow b \\
b \vee c \vee d & \leftarrow
\end{aligned}
$$

$\operatorname{Thus,} \operatorname{can}\left(m s\left(P_{\Delta}^{+}\right)\right)=\{a \vee c \vee d, b \vee c \vee d\}$.

The following definition introduces a special resolution $\vdash_{P}$ which resolves default-negation literals with a disjunction.

Definition 3 Let $\Delta$ be a hypothesis of disjunctive program $P$ and $A \in D B_{P}^{+}$. If there exist $B \in D B_{P}^{+}$and not $b_{1}, \ldots$, not $b_{m} \in \Delta$ such that $B=A \vee b_{1} \vee \cdots \vee b_{m}$ and $B \in \operatorname{can}\left(m s\left(P_{\Delta}^{+}\right)\right)$. Then $\Delta$ is said to be a supporting hypothesis for $A$, denoted $\Delta \vdash_{P} A$.

The set of all positive disjunctions supported by $\Delta$ is denoted:

$$
\operatorname{cons}_{P}(\Delta)=\left\{A \in D B_{P}^{+} \mid \Delta \vdash_{P} A\right\}
$$

Example 3.2 Consider the following disjunctive program $P$ :

$$
\begin{aligned}
a \vee b & \leftarrow c, \text { not } d \\
c \vee e & \leftarrow g, \text { not } f \\
a \vee d & \leftarrow \text { not } b \\
g & \leftarrow
\end{aligned}
$$

Let $\Delta=\{$ not $e$, not $d$, not $f\}$. Then $P_{\Delta}^{+}$consists of the following three rules:

$$
\begin{aligned}
a \vee b & \leftarrow c \\
c \vee e & \leftarrow g \\
g & \leftarrow
\end{aligned}
$$

Thus, $\operatorname{can}\left(m s\left(P_{\Delta}^{+}\right)\right)=\{g, c \vee e, a \vee b \vee e\}$. Since not e is in $\Delta$, we have $\Delta \vdash_{P} a \vee b$.

To specify what is an acceptable hypothesis for a given disjunctive program, some more constraints will be required so that unacceptable hypotheses are ruled out.

Definition 4 Let $\Delta$ and $\Delta^{\prime}$ be two hypotheses of disjunctive program $P$. We say $\Delta$ attacks $\Delta^{\prime}$, denoted $\Delta \leadsto P \Delta^{\prime}$, if at least one of the following two conditions holds:

1. there exists $\beta=$ not $b_{1} \vee \cdots \vee$ not $b_{m} \in \Delta^{\prime}, m>0$, such that $\Delta \vdash_{P} b_{i}$, for all $i=1, \ldots, m$; or

2. there exist not $b_{1}, \ldots$, not $b_{m} \in \Delta^{\prime}, m>0$, such that $\Delta \vdash_{P} b_{1} \vee \cdots \vee b_{m}$,

If $\Delta \sim P \Delta^{\prime}$, we also say $\Delta$ is an attacker of $\Delta^{\prime}$. In particular, if $\Delta \sim_{P}\{n o t p\}$, we simply say that $\Delta$ is an attacker of the assumption not $p$.

Intuitively, $\Delta \sim_{P} \Delta^{\prime}$ means that $\Delta$ causes a direct contradiction with $\Delta^{\prime}$ and the contradiction may come from one of the two cases in Definition 4 


\section{Example 3.3}

$$
\begin{aligned}
a \vee b & \leftarrow \\
c & \leftarrow d, \text { not } a, \text { not } b \\
d & \leftarrow \text { not } e \\
e & \leftarrow \text { t }
\end{aligned}
$$

Let $\Delta^{\prime}=\{$ not $c\}$ and $\Delta=\{$ not $a$, not $b\}$, then $\Delta \sim_{P} \Delta^{\prime}$.

The next definition defines what is an acceptable hypothesis.

Definition 5 Let $\Delta$ be a hypothesis of disjunctive program $P$. An assumption $B$ of $P$ is admissible with respect to $\Delta$ if $\Delta \sim P \Delta^{\prime}$ holds for any hypothesis $\Delta^{\prime}$ of $P$ such that $\Delta^{\prime}{ }_{P}\{B\}$.

Denote $\mathcal{A}_{P}(\Delta)=\left\{\right.$ not $a_{1} \vee \cdots \vee$ not $a_{m} \in D B_{P}^{-} \mid$not $a_{i}$ is admissible wrt $\Delta$ for some $i, 1 \leq$ $i \leq m\}$.

Originally, $\mathcal{A}_{P}$ also includes some other negative disjunctions. To compare with different semantics, we omit them here. Another reason for doing this is that information in form of negative disjunctions does not participate in inferring positive information in DLP.

For any disjunctive program $P, \mathcal{A}_{P}$ is a monotonic operator. Thus $\mathcal{A}_{P}$ has the least fixpoint $\operatorname{lfp}\left(\mathcal{A}_{P}\right)$ and $\operatorname{lfp}\left(\mathcal{A}_{P}\right)=\mathcal{A}_{P}^{k}(\emptyset)$ for some $k \geq 0$ if $P$ is a finite propositional program.

Definition 6 The well-founded disjunctive hypothesis WFDH $(P)$ of disjunctive program $P$ is defined as the least fixpoint of the operator $\mathcal{A}_{P}$. That is, WFDH $(P)=\mathcal{A}_{P} \uparrow \gamma$, where $\gamma$ is an ordinal.

The well-founded disjunctive semantics WFDS for $P$ is defined as the model state $W F D S(P)=W F D H(P) \cup \operatorname{cons}_{P}(W F D H(P))$.

By the above definition, WFDS $(P)$ is uniquely determined by $\operatorname{WFDH}(P)$.

For the disjunctive program $P$ in Example 3.3. $\operatorname{WFDH}(P)=\{n o t c\}$ and $\operatorname{WFDS}(P)=$ $\{a \vee b, d$, not $c\}$. Notice that $e$ is unknown.

A plausible hypothesis should not attack itself.

Definition 7 A hypothesis $\Delta$ is self-attacking if $\Delta \leadsto P \Delta$. Otherwise, we say $\Delta$ is selfconsistent.

It has been proven in [17] that WFDS is consistent in the following sense.

Theorem 3.1 For any disjunctive program $P, W F D H(P)$ is self-consistent and thus WFDS $(P)$ is a consistent model state.

\section{An Alternative Definition of WFDS}

There are several alternative ways of defining argumentative semantics for disjunctive programs and this issue is often confused in literature. In this section, we will try to explain why some of the possible alternatives are unintuitive and then provide an equivalent definition for WFDS. 
One may ask why we cannot replace the inference relation $\vdash_{P}$ with the classical inference relation. This can be clearly explained by the following example. Let $\mathrm{WFDS}_{1}$ denote the disjunctive well-founded semantics obtained by replacing the inference relation $\vdash_{P}$ in Definition 3 with the classical inference relation $\vdash$.

Example 4.1 Let $P$ be the following logic program:

$$
\begin{aligned}
& a \leftarrow \operatorname{not} b \\
& c \leftarrow \operatorname{not} c
\end{aligned}
$$

For this program $P$, its intuitive semantics should be $M_{0}=\{a$, not $b\}$. That is, $a$ is true and $b$ is false while $c$ is undefined. In fact, the well-founded semantics for non-disjunctive logic program assigns the model $M_{0}$ to $P$.

However, if we replace $\vdash_{P}$ with the classical inference relation $\vdash$ in Definition 3 then the resulted disjunctive well-founded semantics $W F D S_{1}$ will derive nothing from $P$, i. e. a, b, c will be all undefined (since $D-W F S_{1}=\emptyset$ ). To see this, let $\Delta^{\prime}=\{$ not $c\}$ and then $P_{\Delta^{\prime}}^{+} \vdash c$. So, $P_{\Delta^{\prime}}^{+} \vdash c \vee b$. Since not $c \in \Delta^{\prime}$, we have $P_{\Delta^{\prime}}^{+} \vdash b$. This means $\{$ not $c\}$ is an attacker of the assumption not $b$. However, $\emptyset$ cannot attack $\{$ not $c\}$.

One might further argue that the unintuitive behavior above of $\mathrm{WFDS}_{1}$ is not caused by replacing $\vdash_{P}$ with the classical inference relation $\vdash$ in Definition 3 but by our allowing self-attacking hypothesis $\Delta^{\prime}=\{$ not $c\}$. So, we might try to require that the attacker $\Delta^{\prime}$ in Definition 5 is self-consistent and denote the resulted semantics as D-WFS ${ }_{2}$. This modification causes an unintended semantics again. For example, let $P$ consist of only one rule $a \vee c \leftarrow$ not $c$. Although $\{$ not $c\} \sim_{P} a$ but $\{$ not $c\}$ is self-attacking. That is, the assumption not $a$ has no self-consistent attacker and thus not $a \in \mathrm{WFDS}_{2}$. This result contradicts to all of the existing well-founded semantics for disjunctive programs.

We have another possibility of modifying Definition 3 Specifically, we can replace $\operatorname{can}\left(\operatorname{ms}\left(P_{\Delta}^{+}\right)\right)$with $T_{P_{\Delta}^{+}}^{S} \uparrow \omega$ and the resulted inference relation is denoted as $\vdash_{P}^{\prime}$. Parallel to Definition 5, we can define a new attack relation $\sim_{P}^{\prime}$ and thus a new disjunctive well-founded semantics denoted D-WFS'.

The inference $\vdash_{P}^{\prime}$ looks more intuitive than $\vdash_{P}$ and in fact we will provide a resolution-like definition for it in the following.

Notice that the inference relation $\vdash_{P}^{\prime}$ is actually a combination of the following two inference rules (the first one is a generalization of the SLI-resolution [11]):

$$
\begin{gathered}
\frac{A \leftarrow a, B, \operatorname{not} C ; \quad a \vee A^{\prime} \leftarrow \operatorname{not} C^{\prime}}{A \vee A^{\prime} \leftarrow B, \operatorname{not} C, \operatorname{not} C^{\prime}} \\
\frac{p_{1} \vee \cdots \vee p_{s} \vee A \leftarrow \operatorname{not} C ; \quad \operatorname{not} C \cup\left\{\operatorname{not} p_{1}, \ldots, \operatorname{not} p_{s}\right\}}{A}
\end{gathered}
$$

Here $A$ is a positive disjunction.

The intuition of rule (4) is quite simple: If we have the hypothesis not $C \cup$ $\left\{\right.$ not $p_{1}, \ldots$, not $\left.p_{s}\right\}$, then we can infer $A$ from the program rule $p_{1} \vee \cdots \vee p_{s} \vee A \leftarrow$ not $C$. Moreover, we can fully perform the rule (3) in advance and then apply the rule (4). 
Since can $\left(\operatorname{ms}\left(P_{\Delta}^{+}\right)\right) \subseteq T_{P_{\Delta}^{+}}^{S} \uparrow \omega$, we have $\Delta \vdash_{P}^{\prime} A$ implies $\Delta \vdash_{P} A$ for any hypothesis $\Delta$ and any disjunction $A$. However, $\vdash_{P}^{\prime}$ is different from $\vdash_{P}$ in general. For instance, let $P=\{a \leftarrow$ $; \quad a \vee b \leftarrow\}$ and $\Delta=\emptyset$. Then $T_{P_{\Delta}^{+}}^{S} \uparrow \omega=\{a, a \vee b\}$ while $\operatorname{can}\left(\operatorname{ms}\left(P_{\Delta}^{+}\right)\right)=\operatorname{can}\left(T_{P_{\Delta}^{+}}^{S} \uparrow \omega\right)=\{a\}$. Even if this fact, the disjunctive well-founded semantics based on these two inference relations become equivalent.

The main result of this section is thus the equivalence of WFDS and WFDS'.

Theorem 4.1 For any disjunctive program $P$, we have

$$
\operatorname{WFDS}^{\prime}(P)=\operatorname{WFDS}(P)
$$

Having this theorem, we will be able to use WFDS to denote both WFDS ${ }^{\prime}$ and WFDS in the following sections.

Proof 4.1 Denote the set of admissible hypotheses for $\Delta$ wrt WFDS $^{\prime}$ as $\mathcal{A}_{P}^{\prime}(\Delta)$ where $P$ is a disjunctive program and $\Delta$ is any hypothesis of $P$.

It suffices to show that

$$
\mathcal{A}_{P} \uparrow \omega=\mathcal{A}_{P}^{\prime} \uparrow \omega .
$$

This is further reduced to proving that

$$
\mathcal{A}_{P} \uparrow k=\mathcal{A}_{P}^{\prime} \uparrow k
$$

for all $k \geq 0$.

We use induction on $k$.

For simplicity, write $\Delta_{k}=\mathcal{A}_{P} \uparrow k$ and $\Delta_{k}^{\prime}=\mathcal{A}_{P}^{\prime} \uparrow k$.

For $k=0$, it is obvious since $\Delta_{0}=\Delta_{0}^{\prime}=\emptyset$.

Assume $\Delta_{k}=\Delta_{k}^{\prime}$, we want to show that $\Delta_{k+1}=\Delta_{k+1}^{\prime}$.

If not $p \in \Delta_{k+1}$, then $\Delta_{k} \leadsto P \Delta^{\prime}$ for any hypothesis $\Delta^{\prime}$ with $\Delta^{\prime} \vdash_{P} p$.

For any hypothesis $\Delta^{\prime}$, if $\Delta^{\prime} \vdash_{P}^{\prime} p$, consider two possible cases:

Case 1. $\Delta^{\prime} \vdash_{P} p$ : then $\Delta_{k} \hookrightarrow_{P} \Delta^{\prime}$ and thus $\Delta_{k} \sim_{P}^{\prime} \Delta^{\prime}$.

Case 2. $\Delta^{\prime} \nvdash_{P} p$ : then the following conditions are satisfied:

(1). $p \notin \operatorname{can}\left(\operatorname{ms}\left(P_{\Delta}^{+}\right)\right)$; and

(2). There is a disjunction $A=a_{1} \vee \cdots \vee a_{m}$ such that $\left\{\right.$ not $a_{1}, \ldots$, not $\left.a_{m}\right\} \subseteq \Delta^{\prime}$, $p \vee A \in T_{P_{\Delta^{\prime}}^{+}}^{S} \uparrow \omega$.

By the above two conditions, there is a sub-disjunction $A^{\prime}$ of $A$ such that $A^{\prime} \in \operatorname{can}\left(\operatorname{ms}\left(P_{\Delta}^{+}\right)\right)$ and $p \notin A^{\prime}$. Thus, $\emptyset \sim_{P} \Delta^{\prime}$. This implies $\Delta \sim_{P} \Delta^{\prime}$. So, $\Delta \sim_{P}^{\prime} \Delta^{\prime}$. That is, not $p \in \Delta_{k+1}^{\prime}$.

For the opposite direction, suppose not $p \in \Delta_{k+1}^{\prime}$. Then $\Delta_{k}^{\prime} \sim_{P}^{\prime} \Delta^{\prime}$ for any hypothesis $\Delta^{\prime}$ with $\Delta^{\prime} \vdash_{P}^{\prime} p$.

For any hypothesis $\Delta^{\prime}$, if $\Delta^{\prime} \vdash_{P} p$, then $\Delta^{\prime} \vdash_{P}^{\prime} p$, which implies $\Delta_{k} \sim_{P}^{\prime} \Delta^{\prime}$ by not $p \in \Delta_{k}^{\prime}$ and the induction assumption. Consider two possible cases:

Case 1. There is an assumption not $a_{1} \vee \cdots \vee$ not $a_{m} \in \Delta^{\prime}$ such that $\Delta_{k} \vdash_{P}^{\prime} a_{i}$ for $1 \leq i \leq m$ : Then for each $a_{i}$, there is a disjunction $a_{i} \vee b_{1} \vee \cdots \vee b_{n} \in T_{P_{\Delta_{k}}^{+}}^{S}$ such that $\left\{\right.$ not $b_{1}, \ldots$, not $\left.b_{n}\right\} \subseteq \Delta_{k}$. If $\Delta_{k} \forall_{P} a_{i}$ for some $i(1 \leq i \leq m)$, then there must be a 
subdisjunction $B^{\prime}$ of $b_{1} \vee \cdots \vee b_{n}$ such that $B^{\prime} \in \operatorname{can}\left(\operatorname{ms}\left(P_{\Delta_{k}}^{+}\right)\right)$. This means $\Delta_{k} \sim_{P} \Delta_{k}$, contradiction.

Therefore, $\Delta_{k} \vdash_{P} a_{i}$ for $1 \leq i \leq m$, which implies $\Delta_{k} \leadsto P \Delta^{\prime}$. Thus, not $p \in \Delta_{k+1}$.

Case 2. There are assumptions not $a_{1}, \ldots$, not $a_{m} \in \Delta^{\prime}$ such that $\Delta^{\prime} \vdash_{P}^{\prime} a_{1} \vee \cdots \vee a_{m}$ where $m>0$ (without loss of generality, we can choose $m$ the least number): Then there is a disjunction $a_{1} \vee \cdots \vee a_{m} \vee b_{1} \vee \cdots \vee b_{n} \in T_{P_{\Delta_{k}}^{+}}^{S} \uparrow \omega$ with not $b_{1}, \ldots$, not $b_{n} \in \Delta_{k}$.

On the contrary, suppose that $\Delta_{k} \nvdash_{P} a_{i}$, then a subdisjunction $B^{\prime}$ of $b_{1} \vee \cdots \vee b_{n}$ is in $\operatorname{can}\left(\operatorname{ms}\left(P_{\Delta_{k}}^{+}\right)\right)$by the minimality of $m$. Thus, we also have $\Delta_{k} \leadsto \Delta_{k}$. This means $\Delta_{k} \vdash_{P} a_{i}$ and therefore, not $p \in \Delta_{k+1}$.

\section{Transformation-based semantics}

As mentioned in Section 1 the transformation-based approach is a promising method of studying semantics for DLP and based on this method, a disjunctive well-founded semantics called D-WFS is defined in [4]. The authors first introduce some intuitive program transformations and then define D-WFS as the weakest semantics that satisfies their transformations. In this section, we shall first analyze the insufficiency of Brass and Dix's set of program transformations and then define a new program transformation called the Elimination of s-implications, which is an extension of a program transformation named the Elimination of nonminimal rules. We then define a new transformation-based semantics, denoted D-WFS*, as the weakest semantics that allows the modified set of program transformations. Our new semantics D-WFS* naturally extends D-WFS and thus is no less skeptical than D-WFS. More importantly, D-WFS* is equivalent to WFDS as we will show in Section 6.

The primary motivation for extending D-WFS is to define a transformation-based counterpart for argumentation-based semantics. However, this extension is also meaningful in view point of commonsense reasoning, because D-WFS seems too skeptical to derive useful information from some disjunctive programs as the next example shows.

Example 5.1 John is traveling in Europe but we are not sure which city he is visiting. We know that, if there is no evidence to show that John is in Paris, he should be either in London or in Berlin. Also, we are informed that John is now visiting either London or Paris. This knowledge base can be conveniently expressed as the following disjunctive logic program $P_{5}$.

$$
\begin{aligned}
& r_{1}: b \vee l \leftarrow \operatorname{not} p \\
& r_{2}: l \vee p \leftarrow
\end{aligned}
$$

Here, $b, l$ and $p$ denote that John is visiting Berlin, London and Paris, respectively.

Intuitively, not b(i.e. John is not visiting Berlin) should be inferred from P. It can be verified that neither $b$ nor its negation not $b$ can be derived from $P$ under D-WFS or STATIC while not $b$ can be derived under WFDS.

Our analysis shows that this unwanted behavior of D-WFS is caused by a program transformation called the Elimination of nonminimal rules [4]: 
- If a rule $r^{\prime}$ is an implication of another rule $r$, then $r^{\prime}$ can be removed from the original program.

According to [4], a rule $r$ is an implication of another rule $r^{\prime}$ if head $\left(r^{\prime}\right) \subseteq \operatorname{head}(r)$, $\operatorname{body}\left(r^{\prime}\right) \subseteq \operatorname{body}(r)$ and at least one inclusion is proper.

This program transformation seems quite intuitive at first glance. For example, if we have a disjunctive program $F_{[}$as follows

$$
\begin{aligned}
r_{1}: b \vee l & \leftarrow \operatorname{not} p \\
r_{3}: \quad l & \leftarrow \operatorname{not} p
\end{aligned}
$$

Then $r_{1}$ is an implication of $r_{3}$ and thus it is intuitive to remove the first rule from $P_{6}$

However, the notion of implication is too weak as shown in Example 5.1. In fact, $r_{2}$ is stronger than $r_{3}$ but $r_{1}$ is not an implication of $r_{2}$ according to Brass and Dix's definition.

Therefore, it is necessary to strengthen the notion of implication so that the application domains as in Example 5.1 can be correctly handled. That is, we want that $r_{1}$ is also an "implication" of $r_{2}$ while $r_{1}$ is an implication of $r_{3}$.

This observation leads to the following strengthening of implication.

Definition $8 r^{\prime}$ is an s-implication of $r$ if $r^{\prime} \neq r$ and at least one of the following two conditions is satisfied:

1. $r^{\prime}$ is an implication of $r$ : head $\left(r^{\prime}\right) \subseteq \operatorname{head}(r), \operatorname{bod} y\left(r^{\prime}\right) \subseteq \operatorname{body}(r)$ and at least one inclusion is proper; or

2. $r$ can be obtained by changing some negative body literals of $r^{\prime}$ into head atoms and removing some body literals from $r^{\prime}$ if necessary.

For instance, according to the second condition in Definition 8 , the rule $b \vee l \leftarrow$ not $p$ is an simplication of the rule $l \vee p \leftarrow$ although $b \vee l \leftarrow$ not $p$ is not an implication of $l \vee p \leftarrow$. It should be pointed out that the notion of s-implications does not mean we transform a disjunctive rule with default negation into a positive rule. Now we prepare to introduce our new transformation-based semantics. According to [4], an abstract semantics can be defined as follows.

Definition 9 A BD-semantics $\mathcal{S}$ is a mapping which assigns to every disjunctive program $P$ a set $\mathcal{S}(P)$ of pure disjunctions such that the following conditions are satisfied:

1. if $Q^{\prime}$ is a sub-disjunction of pure disjunction $Q$ and $Q^{\prime} \in \mathcal{S}(P)$, then $Q \in \mathcal{S}(P)$;

2. if the rule $A \leftarrow$ is in $P$ for a (positive) disjunction $A$, then $A \in \mathcal{S}(P)$;

3. if $a$ is an atom and $a \notin$ head $(P)$ (i. e. a does not appear in the rule heads of $P$ ), then not $a \in \mathcal{S}(P)$. 
In general, a semantics satisfying the above conditions is not necessarily a suitable one because Definition 9 is still very general.

Moreover, as we argued above, it is meaningful to extend the set $\mathbf{T}_{\mathrm{WFS}}$ of program transformations defined in [4]. We accomplish this by introducing a new program transformation called Elimination of s-implications, which extends Brass and Dix's Elimination of nonminimal rules.

The new set $\mathbf{T}_{\mathrm{WFS}}^{*}$ of program transformations is obtained by replacing the Elimination of nonminimal rules in $\mathbf{T}_{\mathrm{WFS}}$ by the Elimination of s-implications (In the sequel, $P_{1}$ and $P_{2}$ are disjunctive programs):

- Unfolding: $P_{2}$ is obtained from $P_{1}$ by unfolding if there is a rule $A \leftarrow b, B$, not $C$ in $P_{1}$ such that

$$
\begin{aligned}
P_{2}= & P_{1}-\{A \leftarrow b, B, \operatorname{not} C\} \\
\cup & \left\{A \vee\left(A^{\prime}-\{b\}\right) \leftarrow B, B^{\prime}, \text { not } C, \text { not } C^{\prime}\right) \mid \\
& \left.\quad \text { there is a rule of } P_{1}: A^{\prime} \leftarrow B^{\prime}, \text { not } C^{\prime} \text { such that } b \in A^{\prime}\right\} .
\end{aligned}
$$

- Elimination of tautologies: $P_{2}$ is obtained from $P_{1}$ by elimination of tautologies if there is a rule $A \leftarrow B$, not $C$ in $P_{1}$ such that $A \cap B \neq \emptyset$ and $P_{2}=P_{1}-\{A \leftarrow B$, not $C\}$.

- Elimination of s-implications: $P_{2}$ is obtained from $P_{1}$ by elimination of s-implications if there are two distinct rules $r$ and $r^{\prime}$ of $P_{1}$ such that $r^{\prime}$ is an s-implication of $r$ and $P_{2}=P_{1}-\left\{r^{\prime}\right\}$.

- Positive reduction: $P_{2}$ is obtained from $P_{1}$ by positive reduction if there is a rule $A \leftarrow$ $B$, not $C$ in $P_{1}$ and $c \in C$ such that $c \notin$ head $\left(P_{1}\right)$ and $P_{2}=P_{1}-\{A \leftarrow B$, not $C\} \cup\{A \leftarrow$ $B, \operatorname{not}(C-\{c\})\}$.

- Negative reduction: $P_{2}$ is obtained from $P_{1}$ by negative reduction if there are two rules $A \leftarrow B$, not $C$ and $A^{\prime} \leftarrow$ in $P_{1}$ such that $A^{\prime} \subseteq C$ and $P_{2}=P_{1}-\{A \leftarrow B$, not $C\}$.

Example 5.2 Consider the disjunctive program $P_{5}$ in Example 5.1 Since $r_{1}$ is an s-implication of $r_{2}, P$ can be transformed into the following disjunctive program $P^{\prime}$ by the Elimination of s-implications:

$$
l \vee p \leftarrow
$$

Suppose that $\mathcal{S}$ is a BD-semantics. Then by Definition $9 \vee p \in \mathcal{S}$ and not $b \in \mathcal{S}$.

Let us consider another example.

Example 5.3 $P$ consists of the following five rules:

$$
\begin{array}{rrrl}
r_{1}: & p \vee p_{1} \vee p_{2} & \leftarrow \\
r_{2}: & p_{1} \vee p_{2} & \leftarrow q \\
r_{3}: & p_{3} & \leftarrow p, q, \text { not } p_{4} \\
r_{4}: & p_{3} \vee p_{4} & \leftarrow \\
r_{5}: & w \vee q & \leftarrow w, \text { not } p \\
r_{6}: & q & \leftarrow
\end{array}
$$

Then we have a transformation sequence: 
1. By the Unfolding, we can remove $q$ from $r_{2}$ and $r_{3}$, and obtain the following $P_{1}$ :

$$
\begin{array}{rrr}
r_{1}: & p \vee p_{1} \vee p_{2} & \leftarrow \\
r_{2}: & p_{1} \vee p_{2} & \leftarrow \\
r_{3}: & p_{3} & \leftarrow p, \text { not } p_{4} \\
r_{4}: & p_{3} \vee p_{4} & \leftarrow \\
r_{5}: & w \vee q & \leftarrow w, \operatorname{not} p \\
r_{6}: & q & \leftarrow
\end{array}
$$

2. By the Elimination of tautologies, we can remove $r_{5}$ and obtain $P_{2}$ :

$$
\begin{array}{rrr}
r_{1}: & p \vee p_{1} \vee p_{2} & \leftarrow \\
r_{2}: & p_{1} \vee p_{2} & \leftarrow \\
r_{3}: & p_{3} & \leftarrow p, \text { not } p_{4} \\
r_{4}: & p_{3} \vee p_{4} & \leftarrow \\
r_{6}: & q & \leftarrow
\end{array}
$$

3. By the Elimination of $s$-implications, we remove $r_{1}$ and obtain $P_{3}$ (since $r_{1}$ is an $s$ implication of $r_{2}$ ):

$$
\begin{array}{rrr}
r_{2}: & p_{1} \vee p_{2} & \leftarrow \\
r_{3}: & p_{3} & \leftarrow p, \text { not } p_{4} \\
r_{4}: & p_{3} \vee p_{4} & \leftarrow \\
r_{6}: & q & \leftarrow
\end{array}
$$

4. Again, by the Elimination of s-implications, we can remove $r_{3}$ and obtain $P_{4}$ (since $r_{3}$ is an s-implication of $\left.r_{4}\right)$ :

$$
\begin{aligned}
& r_{2}: p_{1} \vee p_{2} \leftarrow \\
& r_{4}: p_{3} \vee p_{4} \leftarrow \\
& r_{6}: \quad q \leftarrow
\end{aligned}
$$

5. By the Positive reduction, we obtain

$$
\begin{aligned}
& r_{2}: p_{1} \vee p_{2} \leftarrow \\
& r_{4}: p_{3} \vee p_{4} \leftarrow \\
& r_{6}: \quad q \leftarrow
\end{aligned}
$$

By Definition 9 if $\mathcal{S}$ is a BD-semantics, then $\mathcal{S}(P)$ contains the set of pure literals $\left\{p_{1} \vee p_{2}, p_{3} \vee\right.$ $p_{4}, q$, not $\left.w\right\}$.

We say a semantics $\mathcal{S}$ allows a program transformation $T$ (or equivalently, $\mathcal{S}$ is invariant under $T$ ) if $\mathcal{S}\left(P_{1}\right)=\mathcal{S}\left(P_{2}\right)$ for any two disjunctive programs $P_{1}$ and $P_{2}$ with $P_{2}=T\left(P_{1}\right)$.

Let $\mathcal{S}$ and $\mathcal{S}^{\prime}$ be two BD-semantics. $\mathcal{S}$ is weaker than $\mathcal{S}^{\prime}$ if $\mathcal{S}(P) \subseteq \mathcal{S}^{\prime}(P)$ for any disjunctive program $P$.

We present the main definition of this section as follows. 
Definition $10\left(D-W F S^{*}\right)$ The semantics $D$-WFS for disjunctive programs is defined as the weakest BD-semantics allowing all program transformations in $\mathrm{T}_{W F S}^{*}$.

This definition is not constructive and thus it can not be directly used to compute the semantics D-WFS* and thus a bottom-up procedure will be given in the next section. In the rest of this section, we show some properties of D-WFS*, some of which are generalizations of the corresponding ones for D-WFS given in [4].

We first prove the following two fundamental lemmas.

Lemma 5.1 There is a BD-semantics that allows all the program transformations in $\mathbf{T}_{W F S}^{*}$.

Proof 5.1 We can justify that WFDS is a BD-semantics and allows $\mathrm{T}_{\mathrm{WFS}}^{*}$ (see Proposition 6.1).

Lemma 5.2 Let $\mathrm{C}$ be a non-empty class of BD-semantics for disjunctive programs. Then

1. The intersection $\bigcap_{\mathcal{S} \in \mathrm{C}} \mathcal{S}$ is still a BD-semantics.

2. For any program transformation $T$, if $\mathcal{S}$ allows $T$ for each $\mathcal{S} \in \mathbf{C}$, then $\bigcap_{\mathcal{S} \in \mathbf{C}} \mathcal{S}$ also allows $T$.

The proof of this lemma is direct and thus we omit it here.

Therefore, we have the following result which shows that semantics D-WFS* assigns the unique model state D-WFS* $(P)$ for each disjunctive program $P$.

Theorem 5.1 For any disjunctive program $P, D$-WFS $(P)$ is well-defined.

Proof 5.1 Let Sem $\left(\mathbf{T}_{\mathrm{WFS}}^{*}\right)$ be the class of BD-semantics that allow $\mathbf{T}_{\mathrm{WFS}}^{*}$. Then, by Lemma 5.1 , $\operatorname{Sem}\left(\mathbf{T}_{\mathrm{WFS}}^{*}\right)$ is non-empty. Furthermore, by Lemma 5.2 we have that

$$
\mathrm{D}-\mathrm{WFS}^{*}(P)=\bigcap_{\mathcal{S} \in \operatorname{Sem}\left(\mathbf{T}_{\mathrm{WFS}}^{*}\right)} \mathcal{S}(P)
$$

Since the set $\mathbf{T}_{\mathrm{WFS}}$ of program transformations in [4] is not stronger than $\mathbf{T}_{\mathrm{WFS}}^{*}$, our D-WFS* extends the original D-WFS in the following sense.

Theorem 5.2 Let $P$ be a disjunctive program. Then

$$
D-W F S(P) \subseteq D-W F S^{*}(P) .
$$

The converse of Theorem 5.2 is not true in general. As we will see in Section 6 , for the disjunctive program in Example 5.1 not $b \in \mathrm{D}^{-\mathrm{WFS}^{*}}(P)$ but not $b \notin \mathrm{D}-\mathrm{WFS}(P)$. This theorem also implies that D-WFS* extends the restricted STATIC since the D-WFS is equivalent to the restricted STATIC [5]. 


\section{Bottom-up Computation}

As shown in [4], the transformation-based approach naturally leads to a bottom-up computation for the well-founded semantics. In this section, we will first provide a bottom-up procedure for D-WFS* and then show the equivalence of D-WFS* and WFDS. As a consequence, we also provide a bottom-up computation for WFDS.

Let $P$ be a disjunctive program. Our bottom-up computation for D-WFS* $(P)$ consists of two stages. At the first stage, $P$ is equivalently transformed into a negative program $\operatorname{Lft}(P)$ called the least fixpoint transformation of $P$ [4, 17]. The basic idea is to first evaluate body atoms of the rules in $P$ but delay the negative body literals. The second stage is to further reduce $\operatorname{Lft}(P)$ into another negative disjunctive $\operatorname{res}^{*}(P)$ from which the semantics D-WFS* $(P)$ can be directly read off.

\subsection{The Least Fixpoint Transformation}

In this subsection, we briefly recall the least fixpoint transformation. The details of this notion can be found in 4, 17].

We define the generalized disjunctive base $\mathrm{GDB}_{P}$ of a disjunctive logic program $P$ as the set of all conditional facts whose atoms appear in $P$ :

$$
\begin{aligned}
\mathrm{GDB}_{P}= & \left\{a_{1} \vee \cdots \vee a_{r} \leftarrow \text { not } b_{1}, \ldots, \text { not } b_{s}: a_{i}, b_{j} \in B_{P},\right. \\
& i=1, \ldots, r ; j=1, \ldots, s \text { and } r>0, s \geq 0\}
\end{aligned}
$$

Having the notion of the generalized disjunctive base, we are ready to introduce the immediate consequence operator $T_{P}^{G}$ for general disjunctive program $P$, which generalizes the immediate consequence operator for positive program $P$ (see Definition 11). The definition of the least fixpoint transformation will be based on this operator.

Definition 11 For any disjunctive program $P$, the generalized consequence operator $T_{P}^{G}$ : $2^{G D B_{P}} \rightarrow 2^{G D B_{P}}$ is defined as, for any $J \subseteq G D B_{P}$,

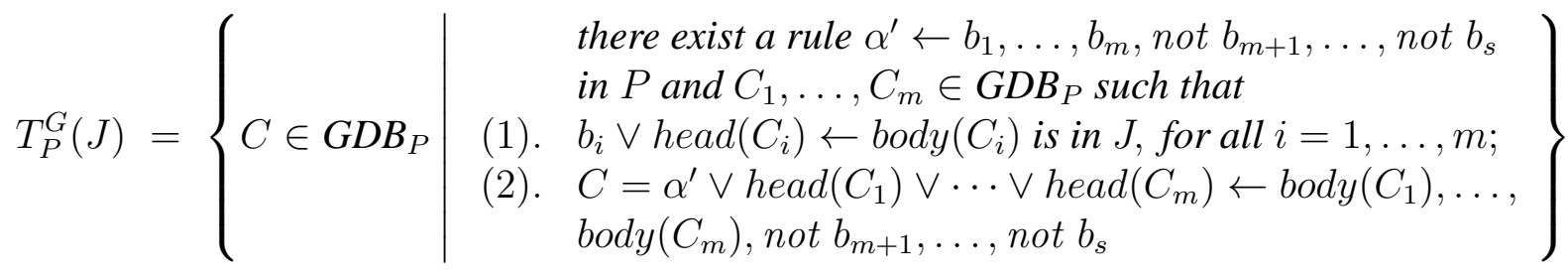

This definition looks a little tedious at first sight. In fact, its intuition is quite simple - it defines the following form of resolution:

$$
\frac{\alpha^{\prime} \leftarrow b_{1}, \ldots, b_{m}, \beta_{1}, \ldots, \beta_{s} ; b_{1} \vee \alpha_{1} \leftarrow \beta_{11}, \ldots, \beta_{1 t_{1}} ; \cdots ; b_{m} \vee \alpha_{m} \leftarrow \beta_{m 1}, \ldots, \beta_{m t_{m}}}{\alpha^{\prime} \vee \alpha_{1} \vee \cdots \vee \alpha_{m} \leftarrow \beta_{11}, \ldots, \beta_{1 t_{1}}, \cdots, \beta_{m 1}, \ldots, \beta_{m t_{m}}, \beta_{1}, \ldots, \beta_{s}}
$$

where $\alpha^{\prime}$ and $\alpha$ s with subscripts are positive disjunctive literals; $\beta$ s with subscripts are negative literals. 
For any disjunctive program $P$, its generalized consequence operator $T_{P}^{G}$ is continuous and hence possesses the least fixpoint $\operatorname{Lft}(P)=T_{P}^{G} \uparrow \omega$. Notice that $\operatorname{Lft}(P)$ is a negative disjunctive program and is said to be the least fixpoint transformation of $P$.

For instance, consider the following disjunctive program $P$ :

$$
\begin{aligned}
b \vee l & \leftarrow u, \text { not } p \\
l & \leftarrow v \\
p \vee v & \leftarrow u, \text { not } w \\
u & \leftarrow
\end{aligned}
$$

Then its least fixpoint transformation $\operatorname{Lft}(P)$ is as follows:

$$
\begin{aligned}
b \vee l & \leftarrow \text { not } p \\
l \vee p & \leftarrow \text { not } w \\
p \vee v & \leftarrow \text { not } w \\
u & \leftarrow
\end{aligned}
$$

Lemma 5.1 in [17] can be restated as the following.

Lemma 6.1 Let $\Delta$ be a hypothesis of disjunctive program $P$ (i. e. $\Delta \subseteq D B_{P}^{-}$) and $\alpha \in D B_{P}^{+}$. Then

$$
\Delta \vdash_{P} \alpha \text { if and only if } \Delta \vdash_{L f t(P)} \alpha \text {. }
$$

By Lemma 6.1, it follows that the least fixpoint transformation Lft is invariant under the semantics WFDS.

Theorem 6.1 Let $L f t(P)$ be the least fixpoint transformation of disjunctive program $P$. Then

$$
\operatorname{WFDS}(\operatorname{Lft}(P))=\operatorname{WFDS}(P) \text {. }
$$

It has been proven in [4] that Lft also is invariant under the transformation-based semantics.

Theorem 6.2 If a BD-semantics $\mathcal{S}$ allows the Unfolding and Elimination of tautologies, then $\mathcal{S}(\operatorname{Lft}(P))=\mathcal{S}(P)$.

By Theorem 6.2, it is direct that the least fixpoint transformation Lft is invariant under the semantics D-WFS*.

Corollary 6.1 Let Lft $(P)$ be the least fixpoint transformation of disjunctive program $P$. Then

$$
D-W F S^{*}(L f t(P))=D-W F S^{*}(P) .
$$




\subsection{Strong Residual Program}

In general, the negative program $\operatorname{Lft}(P)$ can be further simplified by deleting unnecessary rules. This leads to the idea of so-called reductions, which was firstly studied in [6] and then generalized to the case of disjunctive logic programs in 4]. The logic program obtained by fully performing reduction on a disjunctive program $P$ is called the residual program of $P$.

In the following we define the notion of strong residual programs, which is a generalization of Brass and Dix's residual programs.

The strong reduction operator $R^{*}$ is defined as, for any negative program $N$ (i. e. a set of conditional facts),

$$
R^{*}(N)=\left\{A \leftarrow \operatorname{not}(C \cap \operatorname{head}(N)) \mid \begin{array}{l}
\text { there is a rule } r \in N: A \leftarrow \operatorname{not} C \text { such that } \\
r \text { is not an s-implication of } r^{\prime} \neq r \text { for any rule } r^{\prime} \in N
\end{array}\right\}
$$

The intuition behind the above operator is very simple: We first select all minimal rules wrt s-implication and then remove all negative body literals whose atom does not appear in a rule head. Since an implication under Brass and Dix's sense is also an s-implication, we have that $R^{*}(N) \subseteq R(N)$ for any negative program $N$. The strong reduction $R^{*}$ is really different from Brass and Dix's reduction $R$. For example, the reduction of program $F_{5}$ is itself while its strong reduction is $l \vee p \leftarrow$. The reduction operator $R^{*}$ also possesses a more elegant form than the one defined in 4 .

For any disjunctive program $P$, we can first transform it into the negative disjunctive program $\operatorname{Lft}(P)$. Then, fully perform the reduction $R^{*}$ on $\operatorname{Lft}(P)$ to obtain a simplified negative program res $^{*}(P)$ (the strong residual program of $P$ ). The iteration procedure of $R^{*}$ will finally stop in finite steps because $B_{P}$ contains finite number of atoms and the total number of atoms occurring in each $N$ is reduced by $R^{*}$. This procedure is precisely formulated in the next definition, which is in a similar form as Definition 3.4 in [4] (the difference is in that we have a new reduction operator $R^{*}$ here).

Definition 12 (strong residual program) Let $P$ be a disjunctive program. Then we have a sequence of negative programs $\left\{N_{i}\right\}_{i \geq 0}$ with $N_{0}=\operatorname{Lft}(P)$ and $N_{i+1}=R^{*}\left(N_{i}\right)$. If $N_{t}$ is a fixpoint of $R^{*}$, i. e. $N_{t}=R^{*}\left(N_{t}\right)$, then we say $N_{t}$ is the strong residual program of $P$ and denote it as $\operatorname{res}^{*}(P)$.

We first show that the strong residual program is invariant under all the program transformations in $\mathbf{T}_{\mathrm{WFS}}^{*}$. This result actually means that the problem of evaluating $P$ can be reduced to that of evaluating its strong residual program.

Theorem 6.3 Let $P$ and $P^{\prime}$ be two disjunctive programs. If $P$ is transformed into $P^{\prime}$ by a program transformation $T$ in $\mathbf{T}_{W F S}^{*}$, then $\operatorname{res}^{*}(P)=\operatorname{res}^{*}\left(P^{\prime}\right)$.

To prove this theorem, we need the following lemma, which is a reformulation of Lemma 4.1 and 4.2 in 4 .

Lemma 6.2 If $P$ is transformed into $P^{\prime}$ by either the Unfolding or the Elimination of Tautology, then 


\section{1. $P$ and $P^{\prime}$ have the same set of minimal models;}

2. $L f t(P)$ and $L f t\left(P^{\prime}\right)$ contain the same set of minimal conditional facts.

Here, we say a conditional fact $A \leftarrow \operatorname{not} C$ is minimal in a set $N$ of conditional facts if there is no conditional fact $A^{\prime} \leftarrow$ not $C^{\prime}$ in $N$ such that $A^{\prime} \subseteq A, C^{\prime} \subseteq C$ and at least one inclusion is strict.

Proof 6.3

1. If $T$ is either the Unfolding or the Elimination of Tautology, then by Lemma 6.2(1), $P$ and $P^{\prime}$ have the same set of minimal models. Thus, by Lemma 6.2 2), $\operatorname{Lft}(P)$ and $\operatorname{Lft}\left(P^{\prime}\right)$ contain the same set of minimal conditional facts. Thus, $\operatorname{res}^{*}(P)=\operatorname{res}^{*}\left(P^{\prime}\right)$.

2. If $T$ is the Elimination of s-implications, then there are two rules $r$ and $r^{\prime}$ in $P$ such that $r^{\prime}$ is an s-implication of $r$ and $P^{\prime}=P-\left\{r^{\prime}\right\}$.

We show that if $r_{1}^{\prime}$ is a resolvent of $r^{\prime}$ with another rule $r^{\prime \prime}$ by the resolution rule (3), then $r_{1}^{\prime}$ is an s-implication of $r_{1}$ where $r_{1}$ is either $r$ or a resolvent of $r$ with $r^{\prime \prime}$ by (3).

To prove this, we need only to consider the following cases:

(a) Let $r^{\prime}$ is of form $A^{\prime} \vee a \leftarrow \operatorname{body}\left(r^{\prime}\right), r^{\prime \prime}$ is of form head $\left(r^{\prime \prime}\right) \leftarrow a, B$, body ${ }^{-}\left(r^{\prime \prime}\right)$ and $r_{1}^{\prime}$ is obtained by resolving the head atom $a$ of $r^{\prime}$ with the body atom $a$ of $r^{\prime \prime}$. That is, $r_{1}^{\prime}$ is of form $A^{\prime} \vee$ head $\left(r^{\prime \prime}\right) \leftarrow \operatorname{body}\left(r^{\prime}\right), B, \operatorname{bod} y^{-}\left(r^{\prime \prime}\right)$ :

If $a$ does not appear in head $(r)$, then it is obvious that $r_{1}^{\prime}$ is s-implication of $r$; otherwise, we assume that $h e a d(r)$ is of form $A \vee a$ such that $A \subseteq A^{\prime}$.

Notice that the resolvent $r_{1}$ of $r^{\prime \prime}$ with $r$ on $a$ is of form

$$
A \vee \operatorname{head}\left(r^{\prime \prime}\right) \leftarrow \operatorname{body}(r), B, \operatorname{bod} y^{-}\left(r^{\prime \prime}\right)
$$

Therefore, $r_{1}^{\prime}$ an s-implication of $r_{1}$.

(b) Let $r^{\prime}$ is of form head $\left(r^{\prime}\right) \leftarrow b, B^{\prime}$, body $y^{-}\left(r^{\prime}\right), r^{\prime \prime}$ is of form $A^{\prime \prime} \vee b \leftarrow \operatorname{body}\left(r^{\prime \prime}\right)$ and $r_{1}^{\prime}$ is obtained by resolving the body atom $b$ of $r^{\prime}$ with the head atom $b$ of $r^{\prime \prime}$. That is, $r_{1}^{\prime}$ is of form head $\left(r^{\prime}\right) \vee A^{\prime \prime} \leftarrow \operatorname{body} y^{-}\left(r^{\prime \prime}\right), B^{\prime}, b o d y^{-}\left(r^{\prime}\right)$.

If $b$ does not appear in the body of $r$, then it is obvious that $r_{1}^{\prime}$ is an s-implication of $r$; otherwise, assume that $b \in \operatorname{body} y^{+}(r)$.

Notice that the resolvent $r_{1}$ of $r$ with $r^{\prime \prime}$ on $b$ is of form

$$
\operatorname{head}(r) \vee A^{\prime \prime} \leftarrow \operatorname{bod} y^{-}\left(r^{\prime \prime}\right), B, \operatorname{bod} y^{-}(r) .
$$

Therefore, $r_{1}^{\prime}$ an s-implication of $r_{1}$.

Thus, we have that $\operatorname{res}^{*}(P)=\operatorname{res}^{*}\left(P^{\prime}\right)$. 
3. If $T$ is the Positive reduction, then there is a rule $A \leftarrow B$, not $C$ in $P_{1}$ and $c \in C$ such that $c \notin \operatorname{head}\left(P_{1}\right)$ and $P_{2}=P_{1}-\{A \leftarrow B$, not $C\} \cup\{A \leftarrow B$, not $(C-\{c\})\}$.

By the definition of the least fixpoint transformation, the only difference of $\operatorname{Lft}(P)$ from $\operatorname{Lft}\left(P^{\prime}\right)$ is in the rules whose body contain not $c$. Moreover, for each conditional fact $r$ in $\operatorname{Lft}(P)$ of form $A \leftarrow \operatorname{not} C$, not $c$, there is the corresponding conditional fact $A \leftarrow$ not $C$ in $\operatorname{Lft}\left(P^{\prime}\right)$. Thus, by definition of the strong reduction, we have that $\operatorname{Lft}(P)=\operatorname{Lft}\left(P^{\prime}\right)$.

This theorem has the following interesting corollary.

Corollary 6.2 Let $\mathcal{S}$ be a BD-semantics allowing $\mathcal{S}(P)=\mathcal{S}\left(\right.$ res $\left.^{*}(P)\right)$ for all disjunctive program $P$. Then $\mathcal{S}$ allows all program transformations in $\mathbf{T}_{W F S}^{*}$.

Proof 6.2 Suppose that a disjunctive program $P$ is transformed into another disjunctive program $P^{\prime}$. By Theorem 6.3 we have $\operatorname{res}^{*}(P)=\operatorname{res}^{*}\left(P^{\prime}\right)$. Thus,

$$
\mathcal{S}(P)=\mathcal{S}\left(\operatorname{res}^{*}(P)\right)=\mathcal{S}\left(\operatorname{res}^{*}\left(P^{\prime}\right)\right)=\mathcal{S}\left(P^{\prime}\right) .
$$

This corollary implies that, if $\mathcal{S}_{0}$ is a mapping from the set of all strong residual programs to the set of model states and it satisfies all properties in Definition 9 then the mapping defined by $\mathcal{S}(P)=\mathcal{S}\left(\right.$ res $\left.^{*}(P)\right)$ is a BD-semantics.

Before we show the main theorem of this section, we need two lemmas.

Lemma 6.3 Given disjunctive program $P$, we have

$$
D-W F S^{*}\left(\operatorname{res}^{*}(P)\right)=S_{0}^{+}(P) \cup S_{0}^{-}(P)
$$

where

$S_{0}^{+}(P)=\left\{A \in D B_{P}^{+} \mid\right.$rule $A^{\prime} \leftarrow$ is in $r_{e s}^{*}(P)$ for some sub-disjunction $A^{\prime}$ of $\left.A\right\}$

$S_{0}^{-}(P)=\left\{A \in D B_{P}^{-} \mid\right.$if a $\notin$ head $\left(\operatorname{res}^{*}(P)\right)$ for some atom a appearing in $A$. $\}$

Thus, for any disjunctive program $P$, it is an easy task to compute the semantics D-WFS* res $\left.^{*}(P)\right)$ of its strong residual program.

Proof 6.3 Define a mapping $\mathcal{S}_{0}$ from disjunctive programs to model states as follows:

$$
\mathcal{S}_{0}(P)=S_{0}^{+}(P) \cup S_{0}^{-}(P) .
$$

Then $\mathcal{S}_{0}$ is a BD-semantics by Definition 9 and $\mathcal{S}_{0}$ allows $\mathbf{T}_{\text {WFS }}^{*}$ by Corollary 6.2

Since D-WFS ${ }^{*}\left(\operatorname{res}^{*}(P)\right)$ is the least BD-semantics that allows $\mathbf{T}_{\mathrm{WFS}}^{*}$, we have that

$$
\operatorname{D-WFS}^{*}\left(\operatorname{res}^{*}(P)\right) \subseteq \mathcal{S}_{0}\left(\operatorname{res}^{*}(P)\right) .
$$

On the other hand, by Definition 9

$$
\mathcal{S}_{0}\left(\operatorname{res}^{*}(P)\right) \subseteq \mathrm{D}-\mathrm{WFS}^{*}\left(\operatorname{res}^{*}(P)\right) .
$$

Therefore, the conclusion of the lemma is correct.

The next lemma says that $P$ is equivalent to $\operatorname{res}^{*}(P)$ under the semantics D-WFS*. 
Lemma 6.4 For any disjunctive program $P$, we have

$$
D-W_{F}^{*}(P)=D-W F S^{*}\left(\operatorname{res}^{*}(P)\right) .
$$

Proof 6.4 By Corollary6.1, it suffices to prove that the conclusion holds for all negative disjunctive programs. Let $N$ be an arbitrary negative program. We want to show that $N$ is equivalent to $R^{*}(N)$ under the semantics D-WFS* That is, D-WFS $(N)=\mathrm{D}-\mathrm{WFS}^{*}\left(R^{*}(N)\right)$. This can be further reduced to show that $N$ can be transformed into $R^{*}(N)$ by the transformations in $\mathbf{T}_{\mathrm{WFS}}^{*}$.

Notice that $R^{*}(N)$ is obtained from $N$ by removing some rules and/or remove some body atoms. There are two possibilities by the definition of $R^{*}$ :

1. A rule $r \in N$ is removed due to that $r$ is an s-implication of $r^{\prime}$ for some $r^{\prime} \in N$ : This removal can be directly simulated by the Elimination of s-implications;

2. A negative literal not $a$ is removed from the body of a rule $r \in N$ due to $a \notin$ head $(N)$ : This removal can be simulated by the Positive reduction.

Thus, $N$ can be transformed into $R^{*}(N)$ through the transformations in $\mathbf{T}_{\mathrm{WFS}}^{*}$.

The main theorem in this section can be stated as follows, which tell us that the evaluation of $P$ under WFDS can be reduced to that of the strong residual program (the latter is an easy job as we have seen).

Theorem 6.4 For any disjunctive program $P$, we have

$$
D-W F S^{*}(P)=S_{0}^{+}(P) \cup S_{0}^{-}(P)
$$

where

$S_{0}^{+}(P)=\left\{A \in D B_{P}^{+} \mid\right.$rule $A^{\prime} \leftarrow$ is in res ${ }^{*}(P)$ for some sub-disjunction $A^{\prime}$ of $\left.A\right\}$

$S_{0}^{-}(P)=\left\{A \in D B_{P}^{-} \mid\right.$if a $\notin$ head $\left(\right.$ res $\left.^{*}(P)\right)$ for some atom a appearing in $\left.A.\right\}$

Proof 6.4 It follows directly from Lemma 6.3 and 6.4 .

Example 6.1 Consider again the disjunctive program $P_{[5}$ in Example 5.1] The strong residual program res* $(P)$ is as follows:

$$
\begin{aligned}
l & \leftarrow \operatorname{not} p \\
l \vee p & \leftarrow
\end{aligned}
$$

Thus, D-WFS $(P)=\{l \vee p, \text { not } b\}^{2}$.

\footnotetext{
${ }^{2} \mathrm{D}-\mathrm{WFS}^{*}(P)$ should include all pure disjunctions implied by either $l \vee p$ or not $b$. However, the little abusing of notion here simplifies our notation.
} 


\subsection{Equivalence of WFDS and D-WFS*}

Before we present the main theorem of this section, we need some properties of WFDS. First, we can justify that WFDS is a semantics in the sense of Definition 9 Moreover, it possesses the following two important properties.

Proposition 6.1 WFDS allows all program transformations in $\mathbf{T}_{W F S}^{*}$.

This proposition implies that the argumentation-based semantics WFDS is always at least as strong as the transformation-based semantics D-WFS*.

Proof 6.1

1. If $P_{1}$ is transformed into $P_{2}$ by either the Unfolding or Elimination of tautologies, then $\operatorname{Lft}\left(P_{1}\right)=\operatorname{Lft}\left(P_{2}\right)$.

Therefore, $\operatorname{WFDS}\left(P_{1}\right)=\operatorname{WFDS}\left(\operatorname{Lft}\left(P_{1}\right)\right)=\operatorname{WFDS}\left(\operatorname{Lft}\left(P_{2}\right)\right)=\operatorname{WFDS}\left(P_{2}\right)$.

2. If $P_{2}$ is obtained from $P_{1}$ by Positive reduction, then there is a rule $A \leftarrow B$, not $C$ in $P_{1}$ and $c \in C$ such that $c \notin$ head $\left(P_{1}\right)$ and $P_{2}=P_{1}-\{A \leftarrow B, \operatorname{not} C\} \cup\{A \leftarrow B, \operatorname{not}(C-\{c\})\}$.

We need only to show that $\operatorname{WFDS}\left(P_{1}\right)$ and $\operatorname{WFDS}\left(P_{2}\right)$ contain the same set of negative literals. That is, $\operatorname{WFDH}\left(P_{1}\right)=\operatorname{WFDH}\left(P_{2}\right)$. We use induction on $k$ to show

$$
\mathcal{A}_{P_{1}}^{k}(\emptyset)=\mathcal{A}_{P_{2}}^{k}(\emptyset)
$$

for any $k \geq 0$.

For $k=0$, it is obvious.

Assume that (9) holds for $k$, we want to prove (9) also holds for $k+1$.

Notice that not $c$ belongs to both $\operatorname{WFDS}\left(P_{1}\right)$ and $\operatorname{WFDS}\left(P_{2}\right)$. In particular, not $c \in$ $\mathcal{A}_{P_{t}}(\emptyset)$ for $t=1,2$.

Let not $p \in \mathcal{A}_{P_{1}}^{k+1}(\emptyset)$. For any hypothesis $\Delta^{\prime}$ with $\Delta^{\prime} \leadsto P_{2}\{$ not $p\}$, we have that

$$
\left(\Delta^{\prime} \cup\{\text { not } c\}\right) \leadsto P_{1}\{\text { not } p\} .
$$

Thus,

$$
\mathcal{A}_{P_{1}}^{k}(\emptyset) \leadsto P_{1}\left(\Delta^{\prime} \cup\{\text { not } c\}\right) .
$$

By induction,

$$
\mathcal{A}_{P_{2}}^{k}(\emptyset) \leadsto P_{1}\left(\Delta^{\prime} \cup\{\text { not } c\}\right) .
$$

Since not $c \in \mathcal{A}_{P_{2}}^{k}(\emptyset)$, we have $\mathcal{A}_{P_{2}}^{k}(\emptyset) \leadsto P_{2} \Delta^{\prime}$.

Therefore, not $p \in \mathcal{A}_{P_{2}}^{k+1}(\emptyset)$.

This implies

$$
\mathcal{A}_{P_{1}}^{k+1}(\emptyset) \subseteq \mathcal{A}_{P_{2}}^{k+1}(\emptyset)
$$

For the converse inclusion, let not $p \in \mathcal{A}_{P_{2}}^{k+1}(\emptyset)$. For any hypothesis $\Delta^{\prime}$ with $\Delta^{\prime} \leadsto P_{1}$ $\{$ not $p\}$, then $\left(\Delta^{\prime} \cup\{\right.$ not $\left.c\}\right) \leadsto P_{2}\{$ not $p\}$. 
This means $\mathcal{A}_{P_{2}}^{k}(\emptyset) \leadsto P_{1}\left(\Delta^{\prime} \cup\{\right.$ not $\left.c\}\right)$.

Since not $c \in \mathcal{A}_{P_{2}}^{k}(\emptyset)$, we have $\mathcal{A}_{P_{2}}^{k}(\emptyset) \sim P_{1} \Delta^{\prime}$.

That is, not $p \in \mathcal{A}_{P_{1}}^{k+1}(\emptyset)$.

Thus,

$$
\mathcal{A}_{P_{2}}^{k+1}(\emptyset) \subseteq \mathcal{A}_{P_{1}}^{k}(\emptyset)
$$

3. If $P_{2}$ is obtained from $P_{1}$ by the Elimination of s-implications, then there are two possible subcases:

(a) There are two rules $r_{1} \in P_{1}$ of form $A_{1} \vee A_{2} \leftarrow B$, not $C$, not $c_{1}, \ldots$, not $c_{t}$ and $r_{2} \in P_{1}$ of form $A_{1} \vee c_{1} \vee \cdots \vee c_{t} \leftarrow B^{\prime}$, not $C^{\prime}$ such that $B^{\prime} \subseteq B, C^{\prime} \subseteq C$ and $P_{2}=P_{1}-\left\{r_{1}\right\}$ :

It suffices to show that for any two hypotheses $\Delta$ and $\Delta^{\prime}$,

$\Delta \leadsto P_{1} \Delta^{\prime}$ iff $\Delta \leadsto P_{2} \Delta^{\prime}$.

This can be reduced to show that, for any hypothesis $\Delta$ and any positive disjunction A,

$\Delta \vdash_{P_{1}} A$ iff $\Delta \vdash_{P_{2}} A$.

It is direct that $\Delta \vdash_{P_{2}} A$ implies $\Delta \vdash_{P_{1}} A$ since $P_{2} \subseteq P_{1}$.

Let $\Delta \vdash_{P_{1}} A$.

If $r_{1}$ is not involved in the derivation of $\Delta \vdash_{P_{1}} A$, it is trivial that $\Delta \vdash_{P_{2}} A$.

If $r_{1}$ is involved in the derivation of $\Delta \vdash_{P_{1}} A$, then $r_{1}$ must be revolved into a rule $r_{3}$ of form $A_{1} \vee A_{2} \vee A_{3} \leftarrow \operatorname{not} C$, not $C^{\prime}$, not $c_{1}, \ldots$, not $c_{t}$. That is, all body atoms should be resolved with other rules until there is no body atom.

If a head atom of $r_{2}$ is resolved with a body literal of another rule $\bar{r}$, then we get a rule of form

$$
A_{1}^{\prime} \vee A_{2}^{\prime} \vee \operatorname{head}(\bar{r}) \leftarrow \operatorname{not} C^{\prime \prime}, \operatorname{not} c_{1}, \ldots, \operatorname{not} c_{t}
$$

where $A_{1}^{\prime} \subseteq A_{1}$.

On the other hand, if we replace $r_{1}$ with $r_{2}$ in the above derivation, we will get a rule of form

$$
A_{1}^{\prime} \vee \operatorname{head}(\bar{r}) \vee c_{1} \vee \cdots \vee c_{t} \leftarrow \operatorname{not} C^{\prime \prime \prime}
$$

such that $C^{\prime \prime \prime} \subset C^{\prime \prime}$.

If $\Delta$ derives $A$ through the rule (10), then $\left\{\right.$ not $c_{1}, \ldots$, not $\left.c_{t}\right\} \subseteq \Delta$.

Notice that $A_{1}^{\prime} \vee$ head $(\bar{r})$ is a sub-disjunction of $A_{1}^{\prime} \vee A_{2}^{\prime} \vee$ head $(\bar{r})$, thus $\Delta$ can also derives $A$ without $r^{\prime}$ (through $r$ ).

This implies that $\Delta \vdash_{P_{2}} A$.

(b) There are two rules $r$ and $r^{\prime}$ in $P_{1}$ such that head $\left(r^{\prime}\right) \subseteq \operatorname{head}(r), \operatorname{bod} y\left(r^{\prime}\right) \subseteq \operatorname{body}(r)$ and $P_{2}=P_{1}-\left\{r^{\prime}\right\}$ : Similar to Case 1 , we can prove that for any positive disjunction $A$ and any hypothesis $\Delta, \Delta \vdash_{P_{1}} A$ iff $\Delta \vdash_{P_{2}} A$. This implies that $\operatorname{WFDS}\left(P_{1}\right)=$ $\operatorname{WFDS}\left(P_{2}\right)$. 
The next result convinces that the strong residual program $\operatorname{res}^{*}(P)$ of disjunctive program $P$ is equivalent to $P$ w.r.t. the semantics WFDS. Therefore, we can first transform $P$ into $\operatorname{res}^{*}(P)$ and then compute WFDS $\left(\right.$ res $\left.^{*}(P)\right)$.

Proposition 6.2 For any disjunctive program $P$,

$$
\operatorname{WFDS}(P)=\operatorname{WFDS}\left(\operatorname{res}^{*}(P)\right) \text {. }
$$

Proof 6.2 By Lemma 6.1 WFDS $(P)=\operatorname{WFDS}(\operatorname{Lft}(P))$.

Similar to Lemma 6.4, we know that $R^{*}$ can be simulated by $\mathbf{T}_{\mathrm{WFS}}^{*}$.

Thus, WFDS $(\operatorname{Lft}(P))=\operatorname{WFDS}\left(\operatorname{res}^{*}(P)\right)$.

That is, $\operatorname{WFDS}(P)=\operatorname{WFDS}\left(\operatorname{res}^{*}(P)\right)$.

Now we can state the main result of this section, which asserts the equivalence of D-WFS* and WFDS.

Theorem 6.5 For any disjunctive logic program $P$,

$$
W F D S(P)=D-W F S^{*}(P) \text {. }
$$

An important implication of this result is that the well-founded semantics WFDS also enjoys a bottom-up procedure similar to the D-WFS.

Proof 6.5 For simplicity, we denote $\operatorname{res}^{*}(P)$ by $N$ throughout this proof.

By Proposition 6.2 it suffices to show that $\operatorname{WFDS}(N)=\mathrm{D}^{-W_{F}}{ }^{*}(N)$ for any disjunctive program $P$.

First, from Proposition 6.1, it follows that $\operatorname{WFDS}(N) \supseteq \mathrm{D}-W F S^{*}(N)$.

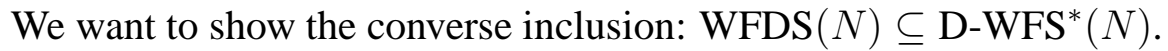

Let $A \in \operatorname{WFDS}(N)$, we consider two cases:

Case 1. $A$ is a negative disjunction: then not $a$ is in $\operatorname{WFDS}(N)$ for some atom $a$ in $A$. It suffices to show that $a \notin h e a d(N)$ for any negative literal not $a$ in $\mathcal{A}_{N}^{k}$ for $k \geq 0$.

We use induction on $k$.

It is obvious for $k=0$.

Assume that the above proposition holds for $k$, we want to show that it also holds for $k+1$.

Let not $a \in \mathcal{A}_{N}^{k+1}$. On the contrary, suppose that there is a rule $r: a \vee A^{\prime} \leftarrow$ not $C^{\prime}$ in $N$. Denote $\Delta^{\prime}=\left\{\right.$ not $\left.p \mid p \in \operatorname{atoms}\left(A^{\prime}\right) \cup C^{\prime}\right\}$, then $\Delta^{\prime} \sim_{N}\{$ not a $\}$. Thus $\mathcal{A}_{N}^{k} \sim_{N} \Delta^{\prime}$. This means that $\mathcal{A}_{N}^{k} \vdash_{N} c_{1} \vee \cdots \vee c_{t}$ for some atoms $c_{1}, \ldots, c_{t}$ appearing in $\Delta^{\prime}$ and $t>0$. Therefore, there is a rule $c_{1} \vee \cdots \vee c_{t} \vee A^{\prime \prime} \leftarrow$ not $C^{\prime \prime}$ such that $\Delta^{\prime \prime} \subseteq \mathcal{A}_{N}^{k}$ where $\Delta^{\prime \prime}=\{$ not $p \mid p \in$ atoms $\left.\left(A^{\prime \prime}\right) \cup C^{\prime \prime}\right\}$. By the induction assumption, $A^{\prime \prime}=\emptyset$; by $N=\operatorname{res}^{*}(P), C^{\prime \prime}=\emptyset$. This contradicts to the fact $N=\operatorname{res}^{*}(P)$. Therefore, $a \notin$ head $(N)$.

Case 2. $A$ is a positive disjunction: then there is a rule in $N: A^{\prime} \vee A^{\prime \prime} \leftarrow \operatorname{not} C^{\prime \prime}$ such that $A^{\prime} \subseteq A$ and $\Delta^{\prime \prime} \subseteq \operatorname{WFDS}(N)$ where $\Delta^{\prime \prime}$ has the same form as in Case 1. Parallel to Case 1, we can prove that $A^{\prime \prime}=\emptyset$ and since $N=\operatorname{res}^{*}(P), C^{\prime \prime}=\emptyset$. This implies that the rule $A^{\prime} \leftarrow$ is in $N$. 


\section{Unfounded Sets}

The first definition of the well-founded model [16] is given in terms of unfounded sets and it has been proven that the notion of unfounded sets constitutes a powerful and intuitive tool of defining semantics for logic programs. This notion has also been generalized to characterize stable semantics for disjunctive logic programs in [7, 10]. However, the two kinds of unfounded sets defined in [7; 10] can not be used to define an intended well-founded semantics for disjunctive programs.

Example 7.1 3

$$
\begin{aligned}
a \vee b & \leftarrow \\
c & \leftarrow \operatorname{not} a, \operatorname{not} b
\end{aligned}
$$

Intuitively, not $c$ should be derived from the above disjunctive program and actually, many semantics including DWFS, STATIC and WFDS assign a truth value 'false' for $c$. However, according to the definitions of unfounded sets in [10, 7], $c$ is not in any $n$-fold application of the well-founded operators on the empty set. For this reason, a more reasonable definition of the unfounded sets for disjunctive programs is in order.

In this section, we will define a new notion of unfounded sets for disjunctive programs and show that the well-founded semantics U-WFS defined by our notion is equivalent to D-WFS* and WFDS.

We say body $(r)$ of $r \in P$ is true wrt model state $\mathrm{S}$, denoted $S \models \operatorname{bod} y(r)$, if $\operatorname{body}(r) \subseteq S$; body $(r)$ is false wrt model state $S$, denoted $S \models \neg$ body $(r)$ if either (1) the complement of a literal in $\operatorname{body}(r)$ is in $S$ or (2) there is a disjunction $a_{1} \vee \cdots \vee a_{n} \in S$ such that $\left\{\right.$ not $a_{1}, \ldots$, not $\left.a_{n}\right\} \subseteq$ $\operatorname{body}(r)$.

In Example 7.1 the body of the second rule is false wrt $S=\{a \vee b\}$.

Definition 13 Let $S$ be a model state of disjunctive program $P$, a set $X$ of ground atoms is an unfounded set for $P$ wrt $S$ if, for each $a \in X$ and each rule $r \in P$ such that $a \in$ head $(r)$, at least one of the following conditions holds:

1. the body of $r$ is false wrt $S$;

2. there is $x \in X$ such that $x \in \operatorname{bod} y^{+}(r)$;

3. if $S \models \operatorname{body}(r)$, then $S \models(h e a d(r)-X)$.

Notice that the above definition generalized the notions of unfounded sets in [7, 10] in two ways. Firstly, the original ones are defined only for interpretations (sets of ground literals) rather than for model states. An interpretation is a model state but not vice versa. Secondly, though one can redefine the original notions of unfounded sets for model states, such unfounded sets are still too weak to capture the intended well-founded semantics of some disjunctive programs. Consider Example 7.1, let $S=\{a \vee b\}$. According to Definition 13, the set $\{c\}$ is an unfounded set of $P$ wrt $S$, but $\{c\}$ is not an unfounded set in the sense of Leone et al or Eiter et al.

Having the new notion of unfounded sets, we are ready to define the well-known operator $\mathcal{W}_{P}$ for any disjunctive program $P$.

\footnotetext{
${ }^{3}$ This example is due to Jürgen Dix (personal communication).
} 
If $P$ has the greatest unfounded set wrt a model state $S$, we denote it $\mathcal{U}_{P}(S)$. However, $\mathcal{U}_{P}(S)$ may be undefined for some $S$. For example, let $P=\{a \vee b\}$ and $S=\{a, b\}$. Then $X_{1}=\{a\}$ and $X_{2}=\{b\}$ are two unfounded sets wrt $S$ but $X=\{a, b\}$ is not.

Definition 14 Let $P$ be a disjunctive program, the operator $\mathcal{T}_{P}$ is defined as, for any model state $S$,

$$
\begin{aligned}
\mathcal{T}_{P}(S)= & \left\{A \in D B_{P} \mid \text { there is a rule } r \in P: A \vee a_{1} \vee \cdots \vee a_{n} \leftarrow \operatorname{body}(r)\right. \text { such that } \\
& \left.S \models \operatorname{body}(r) \text { and not } a_{1}, \ldots, \text { not } a_{n} \in S\right\} .
\end{aligned}
$$

Notice that $\mathcal{T}_{P}(S)$ is a set of positive disjunctions rather than just a set of atoms.

Definition 15 Let $P$ be a disjunctive program, the operator $\mathcal{W}_{P}$ is defined as, for any model state $S$,

$$
\mathcal{W}_{P}(S)=\mathcal{T}_{P}(S) \cup \operatorname{not} . \mathcal{U}_{P}(S)
$$

where not. $\mathcal{U}_{P}(S)=\left\{\right.$ not $\left.p \mid p \in \mathcal{U}_{P}(S)\right\}$.

In general, $\mathcal{W}_{P}$ is a partial function because there may be no greatest unfounded set wrt model state $S$ as mentioned previously.

However, we can prove that $\mathcal{W}_{P}$ has the least fixpoint. Given a disjunctive program $P$, we define a sequence of model states $\left\{W_{k}\right\}_{k \in \mathcal{N}}$ where $W_{0}=\emptyset$ and $W_{k}=\mathcal{W}_{P}\left(W_{k-1}\right)$ for $k>0$.

Similar to Proposition 5.6 in [10], we can prove the following proposition.

Proposition 7.1 Let $P$ be a disjunctive program. Then

1. Every model state $W_{k}$ is well-defined and the sequence $\left\{W_{k}\right\}_{k \in \mathcal{N}}$ is increasing.

2. the limit $\cup_{k \geq 0} W_{k}$ of the sequence $\left\{W_{k}\right\}_{k \in \mathcal{N}}$ is the least fixpoint of $\mathcal{W}_{P}$.

Before proving this proposition, we need the following lemmas.

Lemma 7.1 1. For each $k \geq 0, W_{k}$ is a consistent model state.

2. For any $k \geq 0$ and any unfounded set $X$ for $P$ wrt $W_{k}$, we have $W_{k} \cap X=\emptyset$.

Proof 7.1 We prove this lemma by using simultaneous induction on $k$.

Base The conclusion is obviously true for $k=0$.

\section{Induction}

1. On the contrary, suppose that $W_{k}$ is not a consistent model state. Then there is a positive disjunction $a_{1} \vee \cdots \vee a_{n} \in W_{k}(n \geq 1)$ such that not $a_{i} \in W_{k}$ for any $1 \leq i \leq n$.

Since $a_{1} \vee \cdots \vee a_{n} \in W_{k}=\mathcal{W}\left(W_{k-1}\right)$, there exists a rule $r \in P$ :

$$
a_{1} \vee \cdots \vee a_{n} \vee A \leftarrow \operatorname{body}(r)
$$

such that $W_{k-1} \models \operatorname{body}(r)$ and not $a \in W_{k-1}$ for any $a \in A$. 
By the induction assumption, $W_{k-1}$ is a consistent model state, we know that none of the conditions (1) and (2) in Definition 13 is satisfied by $X=\mathcal{U}\left(W_{k-1}\right)$ wrt $S=W_{k-1}$ and $r$.

Because $X$ is an unfounded set, the condition (3) in Definition 13 should be satisfied by $X=\mathcal{U}\left(W_{k-1}\right)$ wrt $S=W_{k-1}$ and $r$. Thus, $W_{k-1} \models(h e a d(r)-X)$. Note that, since $a_{i} \in X$ for $i=1, \ldots, n$, head $(r)-X=A-X$.

On the other hand, by induction assumption, $W_{k-1}$ is consistent and thus, $W_{k-1} \not \forall(A-X)$ since not $a \in W_{k-1}$ for any $a \in A$, contradiction.

2. On the contrary, suppose that there is an unfounded set $X$ for $P$ wrt $W_{k}$ such that $W_{k} \cap X \neq$ $\emptyset$.

Note that, by induction assumption, $W_{k-1} \cap X=\emptyset$.

Let $a \in W_{k} \cap X$. Then $a \in X$ implies the three conditions in Definition 13 are satisfied by $r$ wrt $S=W_{k}$ and $X$.

On the other hand, by assumption, $a \in W_{k} \backslash W_{k-1}$. Since $a \in W_{k}=\mathcal{T}\left(W_{k-1}\right)$, there exists a rule $r \in P$ such that $W_{k-1} \models \operatorname{body}(r)$ and not $b \in W_{k-1}$ for any $b \in(\operatorname{head}(r)-a)$. This directly implies that none of the conditions (1) and (2) in Definition 13 is satisfied by $r$ wrt $S=W_{k}$ and $X$. Since $W_{k-1} \subseteq W_{k}$, we have $W_{k} \models \operatorname{body}(r)$ and not $b \in W_{k}$ for any $b \in \operatorname{head}(r)-a$.

From the first part of this lemma, it follows that the condition (3) is not satisfied by $r$ wrt $S=W_{k}$ and $X$, contradiction.

Lemma 7.2 Let $S$ be a model state of disjunctive program $P$ such that $S \cap X=\emptyset$.

Then $P$ has the greatest unfounded $\operatorname{set} \mathcal{U}_{P}(S)$.

Proof 7.2 It suffices to prove that the union $U$ of a class $\mathcal{C}$ of unfounded sets for $P$ wrt $S$ is also an unfounded set for $P$ wrt $S$.

For any $a \in U$ and any $r \in P$ such that $a \in \operatorname{head}(r)$, there is an unfounded set $X \in \mathcal{C}$ with $a \in X$. Then there are three possibilities:

1. $\operatorname{body}(r)$ is false wrt $S$;

2. There exists $x \in X$ such that $x \in \operatorname{body} y^{+}(r)$ : It is obvious that $x \in U$;

3. $S \models \operatorname{bod} y(r)$ implies $S \models(h e a d(r)-X)$ :

If $S \models \operatorname{bod} y(r)$, then there is a sub-disjunction $A$ of head $(r)-X$ such that $A \in S$.

Since $U \cap S=\emptyset$, we have that $A \in(\operatorname{head}(r)-U)$.

This means that $U$ is also an unfounded set for $P$ wrt $S$. 
Since we consider only finite propositional programs in this paper, there is some $t \geq 0$ such that $W_{t}=W_{t+1}$.

Having Proposition 7.1 we can define our disjunctive well-founded semantics U-WFS in term of the operator $\mathcal{W}$.

Definition 16 The well-founded semantics $U$-WFS is defined by

$$
U-W F S(P)=\operatorname{lfp}\left(\mathcal{W}_{P}\right) .
$$

For the program $P$ in Example 7.1, U-WFS $(P)=\{a \vee b$, not $c\}$.

An important result is that WFDS is equivalent to U-WFS. This means WFDS and D-WFS* can also be equivalently characterized in term of the unfounded sets defined in this section.

Theorem 7.1 For any disjunctive program $P$,

$$
W F D S(P)=U-W F S(P)
$$

Theorem 7.1 provides further evidence for suitability of WFDS (equivalently, D-WFS*) as the intended well-founded semantics for disjunctive logic programs.

By the following lemma, we can directly prove Theorem 7.1 .

Lemma 7.3 Let $P$ be a disjunctive program. Then $W_{k}=S_{k}$ for any $k \geq 0$.

This lemma also reveals a kind of correspondence between the well-founded disjunctive hypotheses and the unfounded sets.

Proof 7.3 We use induction on $k$ : it is obvious that $S_{0}=W_{0}=\emptyset$. Suppose that $S_{k}=W_{k}$, we want to show that $S_{k+1}=W_{k+1}$. By induction assumption, it suffices to show that $\mathcal{A}_{P}\left(S_{k}^{-}\right)=$ $\operatorname{not}_{P}\left(W_{k}\right)$. This is equivalent to prove that $X_{k}=\mathcal{U}_{P}\left(S_{k}\right)$ where $X_{k}=\left\{p \mid\right.$ not $\left.p \in \mathcal{A}_{P}\left(S_{k}^{-}\right)\right\}$. We prove this statement by the following two steps.

1. $X_{k}$ is an unfounded set of $P$ wrt $S_{k}$ :

Assume that there is a rule $r$ in $P: a_{1} \vee \cdots \vee a_{n} \leftarrow \operatorname{body}(r)$ such that neither the condition 1 nor 2 in Definition 13 is satisfied by $r$.

Without loss of generality, assume that $\left\{a_{1}, \ldots, a_{u}\right\} \subseteq X_{k}$ but $\left\{a_{u+1}, \ldots, a_{n}\right\} \cap X_{k}=\emptyset$.

If $S_{k} \models \operatorname{body}(r)$, then $\Delta^{\prime} \sim_{P}\left\{\right.$ not $a_{1}, \ldots$, not $\left.a_{u}\right\}$ where $\Delta^{\prime}=S_{k}^{-} \cup$ $\left\{\right.$ not $a_{u+1}, \ldots$, not $\left.a_{n}\right\}$.

Notice that $\left\{\right.$ not $a_{1}, \ldots$, not $\left.a_{u}\right\} \subseteq \mathcal{A}_{P}\left(S_{k}^{-}\right)$, it follows that $S_{k}^{-} \sim_{P} \Delta^{\prime}$. Since the hypothesis $S_{k}^{-}$is self-consistent, it should be the case that $\Delta^{\prime} \leadsto_{P}\left\{\right.$ not $a_{u+1}, \ldots$, not $\left.a_{n}\right\}$.

This means that $S_{k}^{-} \vdash_{P} a_{i_{1}} \vee \cdots \vee a_{i_{m}}$ for a subset $\left\{a_{i_{1}}, \cdots, a_{i_{m}}\right\}$ of $\left\{a_{u+1}, \ldots, a_{n}\right\}$.

Thus, $S_{k} \models a_{u+1} \vee \ldots \vee a_{n}$. That is, $S_{k} \models\left(\operatorname{head}(r)-X_{k}\right)$. 
2. $X_{k}$ is the greatest unfounded set of $P$ wrt $S_{k}$ :

We want to prove that each unfounded set $X$ of $P$ wrt $S_{k}$ is a subset of $X_{k}$.

It suffices to show that, for any $a \in X$, not $a$ is acceptable by $\mathcal{A}_{P}\left(S_{k}^{-}\right)$.

Notice that, for any $a \in X$, the set $\{a\}$ is also an unfounded set of $P$ wrt $S_{k}$.

For any hypothesis $\Delta^{\prime}$ such that $\Delta^{\prime} \leadsto_{P}\{$ not a $\}$, then there is a rule $r$ of $P$ :

$$
a \vee a_{1} \vee \cdots \vee a_{n} \leftarrow \operatorname{body}(r)
$$

such that not $a_{i} \in \Delta^{\prime}$ for $i=1, \ldots, n$ and $S_{k}^{-} \vdash_{P} \operatorname{body}(r)$.

On the other hand, since $\{a\}$ is unfounded, we have that $a_{1} \vee \cdots \vee a_{n} \in S_{k}$.

This implies that $S_{k}^{-} \sim_{P}\left\{\right.$ not $a_{1}, \ldots$, not $\left.a_{n}\right\}$. That is, $S_{k}^{-} \sim_{P} \Delta^{\prime}$.

Therefore, not $a \in \mathcal{A}_{P}\left(S_{k}^{-}\right)$.

\section{Conclusion}

In this paper we have investigated recent approaches to defining well-founded semantics for disjunctive logic programs. We first provided a minor modification of the argumentative semantics WFDS defined in [17]. Based on some intuitive program transformations, we proposed an extension D-WFS* to the D-WFS in [4] by introducing a new program transformation called the Elimination of s-implications. This transformation intuitively extends Brass and Dix's two program transformations (Elimination of nonminimal rules and Negative reduction). We have also given a new definition of the unfounded sets for disjunctive programs, which is a generalization of the unfounded sets investigated by [7; 10]. This new notion of unfounded sets fully takes disjunctive information into consideration and provides another interesting characterization for disjunctive well-founded semantics. The main contribution of this paper is the equivalence of U-WFS, D-WFS and WFDS. We have also provided a bottom-up computation for these equivalent semantics. A top-down procedure D-SLS is presented in [18], which is sound and complete with respect to these three semantics. Therefore, the results shown in this paper together with that in [18] show that the following disjunctive well-founded semantics are equivalent:

- D-WFS* based on program transformation;

- WFDS based on argumentation;

- U-WFS based on unfounded sets;

- D-SLS based on resolution.

These results show that, despite diverse proposals on defining disjunctive well-founded semantics, some agreements still exist. The fact that different starting points lead to the same semantics provides a strong support for WFDS (equivalently, D-WFS*, U-WFS and D-SLS). However, it is unclear to us whether these equivalent semantics can be characterized by modifying STATIC [13]. 
Acknowledgments The author would like to thank Philippe Besnard, Alexander Bochman, James Delgrande, Norman Foo, Thomas Linke, Torsten Schaub and Yan Zhang for helpful comments on this work. This work was supported by DFG under grant FOR 375/1-1, TP C. NSFC under grant 69883008.

\section{References}

[1] K. R. Apt, R. N. Bol. Logic programming and negation: A survey. Journal of Logic Programming, 19/20: 9-71.

[2] C. Baral, M. Gelfond. Logic Programming and Knowledge Representation. Journal of Logic Programming, 19/20: 73-148.

[3] S. Brass, J. Dix. Characterizations of the Disjunctive Well-founded Semantics: Confluent Calculi and Iterated GCWA. Journal of Automated Reasoning, 20(1):143-165, 1998.

[4] S. Brass, J. Dix. Semantics of disjunctive logic programs based on partial evaluation. Journal of Logic programming, 38(3):167-312, 1999.

[5] S. Brass, J. Dix, I. Niemelä, T. Przymusinski. On the equivalence of the Static and Disjunctive Well-founded Semantics and its computation. Theoretical Computer Science, 258(1-2): 523-553, 2001.

[6] F. Bry. Negation in logic programming: A formalization in constructive logic. In: D. Karagiannis ed. Information Systems and Artificial Intelligence: Integration Aspects (LNCS 474), Springer, pages 30-46, 1990.

[7] T. Eiter, N. Leone and D. Sacca. On the partial semantics for disjunctive deductive databases. Annals of Math. and AI., 19(1-2): 59-96, 1997.

[8] M. Gelfond, V. Lifschitz. The stable model semantics for logic programming. In: Proceedings of the 5th Symposium on Logic Programming, MIT Press, pages 1070-1080, 1988.

[9] M. Gelfond, V. Lifschitz. Classical negation in logic programs and disjunctive databases. New Generation Computing, 9: 365-385, 1991.

[10] N. Leone, P. Rullo and F. Scarcello. Disjunctive stable models: unfounded sets, fixpoint semantics, and computation. Information and Computation, 135(2): 69-112, 1997.

[11] J. Lobo, J. Minker and A. Rajasekar. Foundations of Disjunctive Logic Programming. MIT Press, 1992.

[12] J. Minker. On indefinite databases and the closed world assumption. LNCS 138, pages 292-308, 1982. 
[13] T. Przymusinski. Static semantics of logic programs. Annals of Math. and AI., 14: 323-357, 1995.

[14] K. Ross. A procedural semantics for well-founded negation in logic programs. Journal of Logic programming, 13(1): 1-22, 1992.

[15] D. Saccá, C. Zaniolo. Stable models and non-determinism in logic programs. Proceedings of the 9th ACM PODS, pages 205-217, 1990.

[16] A. Van Gelder, K. A. Ross and J. Schlipf. The well-founded semantics for general logic programs. J. ACM, 38(3): 620-650, 1991.

[17] K. Wang. Argumentation-based abduction in disjunctive logic programming. Journal of Logic programming, 45(1-3): 105-141, 2000.

[18] K. Wang. A top-down procedure for disjunctive well-founded semantics. In: Proceedings of the International Joint Conference on Automated Reasoning (IJCAR'01), Springer, 2001.

[19] J. You, L. Yuan and R. Goebel. An abductive approach to disjunctive logic programming. Journal of Logic programming, 44(1-3): 101-127, 2000. 